Draft Version MAY 3, 2012

Preprint typeset using LATEX style emulateapj v. 08/13/06

\title{
OBSERVING SIMULATED PROTOSTARS WITH OUTFLOWS: HOW ACCURATE ARE PROTOSTELLAR PROPERTIES INFERRED FROM SEDS?
}

\author{
Stella S. R. OfFner \\ Harvard-Smithsonian Center for Astrophysics, Cambridge, MA 02138, USA
}

Thomas P. Robitaille

Max-Planck-Institute for Astronomy, Königstuhl 17, 69117 Heidelberg, Germany

Charles E. HANSEN

Astronomy Department, UC Berkeley, Berkeley, CA 94720, USA

Christopher F. MCKeE

Physics and Astronomy Departments, UC Berkeley, Berkeley, CA 94720, USA

RichaRd I. KLEIN

Astronomy Department, UC Berkeley, Berkeley, CA 94720; Lawrence Livermore National Laboratory, Livermore, CA 94550, USA

Draft version May 3, 2012

\begin{abstract}
The properties of unresolved protostars and their local environment are frequently inferred from spectral energy distributions (SEDs) using radiative transfer modeling. In this paper, we use synthetic observations of realistic star formation simulations to evaluate the accuracy of properties inferred from fitting model SEDs to observations. We use ORION, an adaptive mesh refinement (AMR) three-dimensional gravito-radiation-hydrodynamics code, to simulate low-mass star formation in a turbulent molecular cloud including the effects of protostellar outflows. To obtain the dust temperature distribution and SEDs of the forming protostars, we post-process the simulations using HYPERION, a state-of-the-art Monte-Carlo radiative transfer code. We find that the ORION and HYPERION dust temperatures typically agree within a factor of two. We compare synthetic SEDs of embedded protostars for a range of evolutionary times, simulation resolutions, aperture sizes, and viewing angles. We demonstrate that complex, asymmetric gas morphology leads to a variety of classifications for individual objects as a function of viewing angle. We derive best-fit source parameters for each SED through comparison with a pre-computed grid of radiative transfer models. While the SED models correctly identify the evolutionary stage of the synthetic sources as embedded protostars, we show that the disk and stellar parameters can be very discrepant from the simulated values, which is expected since the disk and central source are obscured by the protostellar envelope. Parameters such as the stellar accretion rate, stellar mass, and disk mass show better agreement, but can still deviate significantly, and the agreement may in some cases be artificially good due to the limited range of parameters in the set of model SEDs. Lack of correlation between the model and simulation properties in many individual instances cautions against over-interpreting properties inferred from SEDs for unresolved protostellar sources.
\end{abstract}

Subject headings: stars: formation, stars:low-mass

\section{INTRODUCTION}

Young protostars are deeply embedded in molecular gas and dust, which leads to significant reprocessing of their radiation. Since most of these sources cannot be directly imaged, it is necessary to infer properties of the protostar, accretion disk, and gas envelope using other means. The proliferation of infrared and millimeter instruments, such as Spitzer, 2MASS, CARMA, Plateau de Bure, and Herschel, has contributed a wealth of multiwavelength data for numerous embedded protostars over the last decade. Spectral energy distributions (SEDs), generally spanning $\sim 1-1000 \mu \mathrm{m}$, can be constructed from this multiwavelength photometry. However, even with a

Electronic address: soffner@cfa.harvard.edu reasonably complete SED, complications such as excess extinction, parameter degeneracies, and fitting subtleties elevate the derivation of source attributes to an art form.

SEDs are typically divided into four classes using either the infrared spectral slope (Greene et al. 1994) or the effective source ("bolometric") temperature (Myers \& Ladd 1993). These classes generally serve as a proxy for the amount of radiation reprocessing that occurs due to the embedding dense gas. Regardless of the details of the classification scheme, objects characterized as Class 0 protostars are heavily obscured by dusty envelopes such that most of the radiation falls in the sub-millimeter. Class I protostars are less obscured and may be surrounded by a massive circumstellar accretion disk. For a Class II object, some of the direct stellar radiation is vis- 


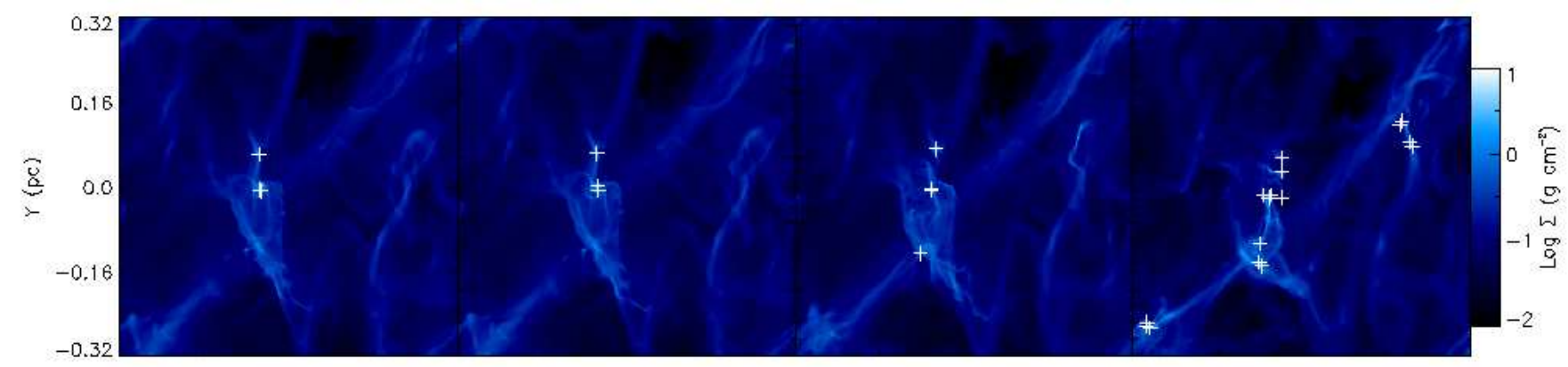

FIG. 1. - Log column density for the 0, 15, 30 and $60 \mathrm{kyr}$ zooms from left to right. Star locations are indicated by the white crosses.

ible, and often the source is considered to be a pre-mainsequence star, which has accreted or expelled most of its initial envelope and thus produces little (sub)millimeter emission. The remaining gas lies in a thin circumstellar accretion disk. Outflow activity may affect the SED shape most significantly during the Class I and Class II phases. For Class III objects, the disk dissipates and the source approaches the main sequence.

While SED classification may be straightforward, deriving actual physical properties from the SEDs is more complicated. For reasons discussed below, it is very difficult to assign more than a rough evolutionary state to a given SED. The most common means of inferring properties involves picking a simple analytic model for the protostellar properties (e.g., mass, radius, and accretion rate) and embedding gas morphology (e.g., disk mass, envelope mass, radial profile, outflow cavity width) and then computing SEDs with a radiative transfer code. The studies published by Robitaille et al. (2006, henceforth R06) and Robitaille et al. (2007), greatly streamlined parameter derivation by providing an extensive library of 200,000 aperture-dependent model SEDs defined by 14 physical parameters (including a wide range of stellar masses and evolutionary stages) and 10 viewing angles for each physical model. The studies also included an efficient way to fit these models to observations and to derive ranges of parameter values providing a good fit. These models have been widely used on problems ranging from estimations of individual stellar properties (Robitaille et al. 2007) to the Milky Way star formation rate (Robitaille \& Whitney 2010).

Despite the breadth of parameter space, the R06 models have limits. The morphologies are axisymmetric and thus do not account for gas turbulence, filamentary structure, or outflow asymmetry. The stellar age is taken as one parameter input, but the models do not form an evolutionary sequence. (Indeed, the detailed evolutionary progression of an individual protostar, including its formation time (McKee \& Offner 2010), accretion history (Offner \& McKee 2011) and their subsequent effects on stellar evolution (Hosokawa et al. 2011) remains debated!). Because of the inherent observational uncertainty in age estimations and parameter degeneracies, SED classes cannot be confidently mapped to stages of evolution (e.g., Crapsi et al. 2008). The R06 models also neglect gas with temperatures below $30 \mathrm{~K}$, which may affect the fits at longer wavelengths. Finally, the models assume that the source is a single protostar. Although most low-mass stars in the field are single (Lada 2006), evidence suggest that protostars may have higher multiplicity fractions (Duchêne et al. 2007). In addition, estimating protostellar multiplicity is challenging even using high-resolution interferometry of local star forming regions (Offner et al. 2011a). In regions more than a kpc distant, where each source is potentially a cluster of young protostars, the multiplicity is impossible to obtain accurately (e.g., Kang et al. 2009).

There are also a number of complications to parameter derivations that are independent of underlying model assumptions. First, there is sizable parameter degeneracy. This can be taken into account by considering the group of models with reasonable fits, i.e., "good-fit" models, rather than any single best-fit model. Second, the source viewing angle has a significant effect on the source classification. Even including an outflow cavity inclination variable, it is very difficult to distinguish an older edge-on Class II source from a younger Class I source or a Class I source viewed along the outflow axis from an older, less embedded Class II source. This type of confusion can be reduced by making mass envelope estimates from observations of the millimeter continuum (Enoch et al. 2009) or by detecting molecular emission associated with dense gas (e.g., Heiderman et al. 2010; van Kempen et al. 2009), however, this data is not always available.

The goal of this work is to model the SEDs of sources in realistic, non-magnetized simulations and explore the challenges of parameter estimation from SEDs based on idealized models (e.g., R06). We use ORION, an adaptive mesh refinement (AMR) three-dimensional gravitoradiation-hydrodynamics code, to simulate low-mass star formation in a turbulent, clustered environment. The simulations include both the effects of radiation feedback from the forming stars and protostellar outflows. In order to obtain model dust temperatures and synthetic SEDs for the sources, we post-process the simulations using the HYPERION radiative transfer code (Robitaille 2011). Section 2 contains an overview of our numerical methods. In section 3 we present the SEDs for four evolutionary times and a variety of simulation resolutions, viewing angles and aperture sizes. We also derive bestfit source parameters for each SED through comparison with the R06 grid of models and then compare these with the actual simulation values. We conclude in section 5 .

\section{METHODS}

\subsection{D Radiation-Hydrodynamic Simulations}




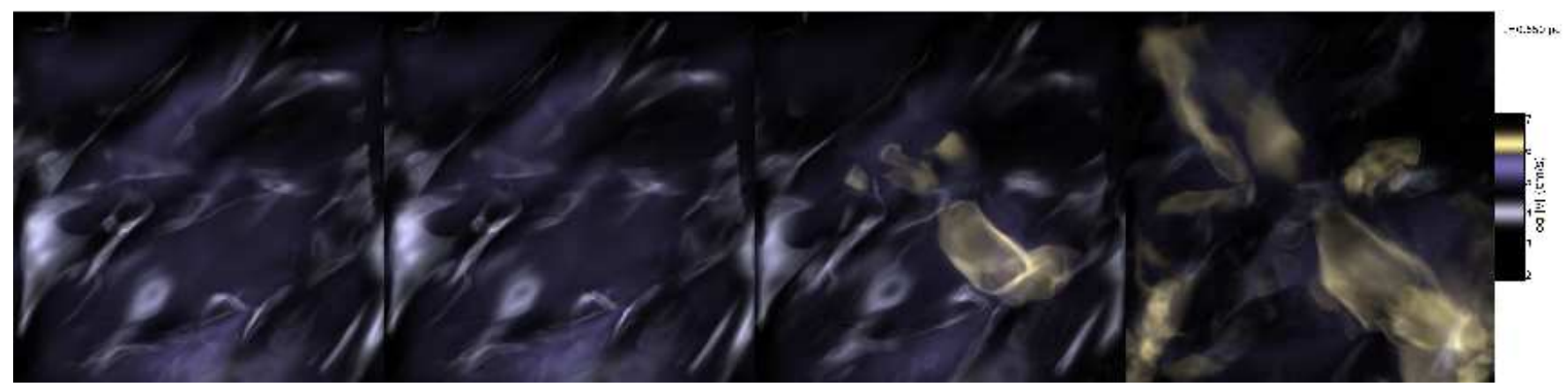

FIG. 2.- Volume rendering of gas velocity for the $0,15,30$ and $60 \mathrm{kyr}$ zooms from left to right.

We perform self-gravitating radiation-hydrodynamic calculations using the ORION Adaptive Mesh Refinement (AMR) code (Truelove et al. 1998; Klein 1999). Our simulations adopt the same initial conditions as Offner et al. (2009) and numerical procedure as Hansen et al. (2012), which we briefly summarize below.

The simulations are of a turbulent box with a mean density of $4.46 \times 10^{-20} \mathrm{~g} \mathrm{~cm}^{-3}$, a total gas mass of 185 $\mathrm{M}_{\odot}$, cloud length of $0.65 \mathrm{pc}$, and an initial 3D Mach number of 6.6. To initialize the domain, we inject energy at a constant rate for two shock crossing times without self-gravity (e.g., following Stone et al. 1998). After this turbulent driving phase, gravity is turned on and the turbulence is allowed to naturally decay.

Sink particles are inserted in regions of the flow that exceed the Jeans condition (Krumholz et al. 2004). These "stars" are endowed with a sub-grid stellar evolution model that includes accretion luminosity down to the stellar surface, Kelvin-Helmholz contraction, and nuclear burning. A second sub-grid model based upon Matzner \& McKee (1999) governs the launching of protostellar winds. Our sub-grid models allows us to include all radiative heating in a way that is independent of the simulation resolution. Since we also include the effects of protostellar outflows, the accretion rate from the disk onto the star is reduced from the case without outflows, and the accretion luminosity is thus smaller than computed in Offner et al. (2009) by a factor of $\sim 10$ (Hansen et al. 2012). As pointed out by Bate (2011), one defect of stellar sub-grid models is that the luminosity depends explicitly on the protostellar radius. This radius can vary by a factor of two depending upon the details of the adopted evolutionary model, including the assumed radiative efficiency, initial seed radius or mass, and gas opacities. Nonetheless, this uncertainty corresponds to a factor of only $2^{1 / 4}(<20 \%)$ in gas temperature (Offner et al. 2009).

We summarize the key aspects of our outflow model below but refer the reader to Cunningham et al. (2011) for the full details of the model implementation in ORION. In our outflow model, the wind launching velocity is given by the Keplerian velocity at the stellar surface, $v_{K}=\sqrt{G M_{*} / r_{*}}$. For high-mass protostars these velocities can exceed $200 \mathrm{~km} \mathrm{~s}^{-1}$, greatly limiting the numerical time step of the calculation. Cunningham et al. (2011) circumvent this issue by capping the outflow velocity at a fixed fraction of the Keplerian speed. In the calculations we present here, we instead economize the computational expense by capping the launching velocity, $v_{K} \leq 60 \mathrm{~km}$ $\mathrm{s}^{-1}$, and only proceeding to high resolution at discrete times.

Note that in our wind model the outflow momentum and direction are determined by the instantaneous protostellar mass, accretion rate, and angular momentum vector. Once deposited on the grid, the gas evolves hydrodynamically, which leads to outflow morphology and asymmetry similar to that of observed outflows (Offner et al. 2011b).

For the dust opacities, we adopt a dust model that assumes a standard iron abundance and treats the grains as composite aggregates (Semenov et al. 2003). This model produces an extinction ratio of $R_{v}=3.42$ for dust with temperature $T<120 \mathrm{~K}$. In most of the domain, dust serves as the dominant coolant. However, strong shocks produced by outflowing gas running into ambient material can result in cells with temperatures exceeding the dust destruction temperature of $\sim 1000 \mathrm{~K}$. In this regime, we implicitly calculate the gas temperatures assuming atomic line cooling (Cunningham et al. 2011).

We perform the numerical calculations as follows. First, we run a simulation of a forming cluster for one freefall time with a maximum cell resolution of $128 \mathrm{AU}$ (two AMR levels). We then "zoom" in on four different time slices by restarting the calculation and allowing it to evolve with five additional AMR levels (4 AU resolution). This is similar to the procedure in Offner et al. (2011b) except that here all protostars receive extra resolution. New grid cells are added to satisfy a Jeans condition with $J=0.125$ (Truelove et al. 1997) and a geometric refinement criterion requiring that each star is centered within a block of $8^{3}$ fine cells. We refer to the high resolution calculations according to the time when the zoom begins relative to the formation of the first star in the simulation: 0 kyr, $15 \mathrm{kyr}, 30 \mathrm{kyr}$ and $60 \mathrm{kyr}$. Extra resolution is added to the first time slice beginning just prior to the formation of the first star particle. During each zoom, we evolve the calculation for $\sim 8 \mathrm{kyr}$, where we allow structure to develop in the newest cells before proceeding to the next AMR level. Table 1 gives the protostellar masses and luminosities at the highest resolution during each of the zooms. In our analysis, the oldest star is $\sim 70$ kyr, so we consider only the earliest embedded stage of star formation in this work.

Figure 1 shows the column density at the completion of each zoom. At the final time, there are 20 stars, which are clustered in several different regions of the domain. Figure 2 shows a volume rendering of the gas velocity for each of the zooms. As the calculation evolves, the outflows of the first forming stars extend across the domain 
TABLE 1

StAR PARTICLE PROPERTIES AT THE HIGHEST RESOLUTION FOR EACH OF THE ZOOMS. THE STARS ARE LISTED IN ORDER OF FORMATION.

\begin{tabular}{|c|c|c|c|}
\hline Time (kyr) & Mass $\left(\mathrm{M}_{\odot}\right)$ & Luminosity $\left(\mathrm{L}_{\odot}\right)$ & Name $^{a}$ \\
\hline \multirow[t]{3}{*}{0} & 0.032 & 0.11 & $1 \mathrm{a}$ \\
\hline & $2.0 \mathrm{e}-6$ & - & \\
\hline & $7.8 \mathrm{e}-5$ & - & \\
\hline \multirow[t]{4}{*}{15} & 0.053 & 0.25 & $1 b$ \\
\hline & 0.023 & 1.06 & $2 \mathrm{a}$ \\
\hline & $1.2 \mathrm{e}-5$ & - & \\
\hline & 0.012 & 0.23 & $3 a$ \\
\hline \multirow[t]{4}{*}{30} & 0.19 & 3.19 & \\
\hline & 0.14 & 1.50 & $2 \mathrm{~b}$ \\
\hline & 0.64 & 1.86 & $1 \mathrm{c}$ \\
\hline & 0.05 & 0.23 & $3 \mathrm{~b}$ \\
\hline \multirow[t]{20}{*}{60} & 0.73 & $77.71^{b}$ & \\
\hline & 0.29 & 0.35 & $2 \mathrm{c}$ \\
\hline & 1.14 & 10.66 & $1 d$ \\
\hline & 0.12 & 1.11 & $3 c$ \\
\hline & 0.13 & 0.26 & 4 \\
\hline & 0.11 & 0.36 & 5 \\
\hline & 0.17 & 1.059 & \\
\hline & 0.065 & 0.24 & 6 \\
\hline & 0.058 & 0.13 & 7 \\
\hline & 0.10 & 1.15 & 8 \\
\hline & 0.52 & 7.39 & 9 \\
\hline & 0.078 & 0.13 & \\
\hline & 0.077 & 0.18 & 10 \\
\hline & 0.047 & 0.20 & 11 \\
\hline & 0.19 & 1.86 & 12 \\
\hline & 0.10 & 0.21 & \\
\hline & 0.038 & 0.11 & 13 \\
\hline & 0.037 & 0.21 & 14 \\
\hline & 0.052 & 0.25 & 15 \\
\hline & 0.036 & 0.13 & 16 \\
\hline
\end{tabular}

a See Figure 6. Only those with sufficient luminosity and those that do not have a nearby, more massive companion are assigned a unique name.

${ }^{b}$ This source is brighter than its more massive companion (1d) because its instanteous accretion rate is higher.

and dominate the turbulent motions. By the final time, the outflows are too entangled to separate individually, and the simulation resembles an outflow dominated cluster like NGC1333 (Swift \& Welch 2008).

\subsection{Spectral Energy Distribution Modeling}

We use HYPERION, a parallel 3D Monte-Carlo dust continuum radiative transfer code, to compute synthetic spectral energy distributions for the forming stars in the simulations (see Robitaille 2011 for algorithmic details). Since the HYPERION infrastructure is designed to be as generic as possible, we are able to map the ORION AMR cells to a tree structure that can be directly read by HYPERION without interpolation or information loss. Moreover, the sources do not need to be excised from the domain and computed separately. For each time slice, HYPERION calculates the SED for a set of apertures centered on each specified source as viewed through the box. In some cases, especially for the larger apertures, multiple sources may contribute to the net emission. The HYPERION inputs are the dust density as a function of position, an opacity model, source luminosities and source temperatures. The dust temperature is calculated by HYPERION based upon the input sources. For consistency, we use the same aggregate dust grain opacity model used in the ORION simulations and adopt the coefficients calculated for dust with temperature $<120 \mathrm{~K}$
(Semenov et al. 2003).

When performing the radiative transfer calculation, we exclude any star particles that have not yet begun burning Deuterium. These star particles tend to have such small masses that their accretion luminosity is negligible. (These stars are shown in Figure 1 for completeness.) We also zero the density within $8^{3}$ fine cells immediately around each star. This volume contains the accretion and outflow launching regions. Numerical effects reduce the accuracy of these cells, so that they may be overdense above and below the disk plane, where the wind would otherwise sweep out a cavity. This is discussed in more detail in section 4.9 and Appendix A.

We perform the radiative transfer calculations with $10^{7}$ photon packets to ensure that the shorter wavelength fluxes are reasonably well converged. For this number, additional photon packets do not significantly change the SED. The SEDs are computed with 200 wavelengths logarithmically distributed between $0.1 \mu \mathrm{m}$ and $5000 \mu \mathrm{m}$. For each source and viewing angle, we compute the flux for 20 circular apertures with radii logarithmically spaced between $1000 \mathrm{AU}$ and 20,000 AU. Each source is viewed from 20 perspectives, which are regularly spaced in $\theta$ and $\phi^{1}$.

\section{SYNTHETIC OBSERVATIONS}

\subsection{Dust Temperature Comparison}

HYPERION calculates the dust temperature, $T$, by assuming local thermodynamic equilibrium (LTE), wherein the dust emission balances the specific energy absorption rate, $\dot{A}$ :

$$
4 \pi \kappa_{\mathrm{P}}(T) B(T)=\dot{A}
$$

where $\kappa_{\mathrm{P}}(T)$ is the pre-computed Planck mean mass absorption coefficient and $B(T)$ is the integrated Planck function. The right side, which depends upon the mean intensity, $J_{\nu}$, can be expressed as a function of the energy per photon packet, $\epsilon$, cell volume, $V$, emission time, $\Delta t$, photon path length between events, $l$, and mass absorption coefficient (Lucy 1999):

$$
\dot{A}=4 \pi \int_{0}^{\infty} \kappa_{\nu} J_{\nu} d \nu=\frac{1}{\Delta t} \frac{\epsilon}{V} \sum l \kappa_{\nu} .
$$

On top of this solution, we impose a minimum dust temperature of $10 \mathrm{~K}$. For HYPERION, stars are the only heating source. Dynamical effects such as shock and viscous heating are neglected by eq. 1. Viscous heating is much smaller than radiative heating, where any significant contribution falls mainly within the disks (Offner et al. 2009). However, shocks may significantly heat the gas, particularly affecting outflow gas more distant from the star.

In comparison, ORION makes several different assumptions in calculating the gas temperature. First, it assumes that the dust and gas are perfectly collisionally coupled and, hence, have the same temperature and velocity. ORION assumes that dust is the primary coolant (except in superheated regions in which the dust has sublimated). To obtain the temperature, ORION solves the

\footnotetext{
1 Geometrically, 20 regular angular spacings in spherical coordinates correspond to the vertexes of a dodecahedron.
} 


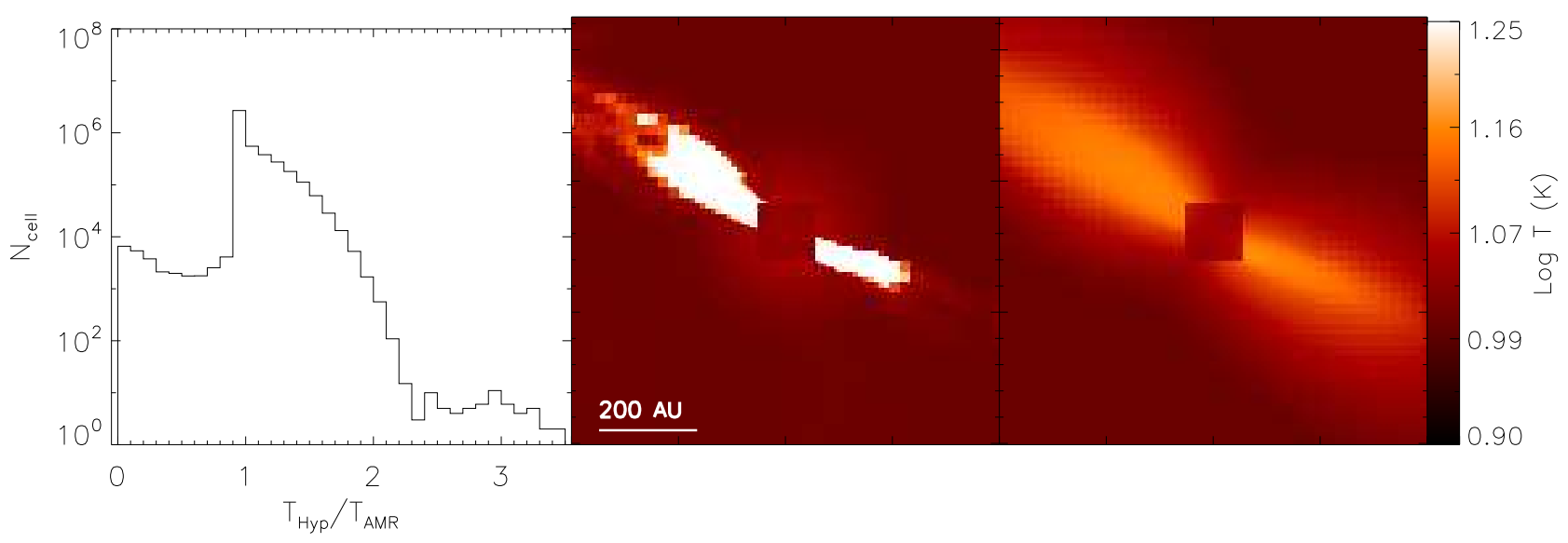

FIG. 3.- Estimated dust temperature for the first forming protostar at 0 kyr (just after the formation of the first sink particle). Left: Histogram of the temperature ratios for all cells in a $(1400 \mathrm{AU})^{3}$ region centered on the protostar. Center: Temperature slice showing the ORION dust temperatures. Right: Temperature slice showing the HYPERION dust temperatures. The central $16^{3}$ finest cells have been excised (this is where the HYPERION density input was set to zero).

non-equilibrium, flux-limited, radiation diffusion equation:

$$
\frac{\partial E}{\partial t}-\nabla \cdot\left(\frac{c \lambda}{\kappa_{\mathrm{R}} \rho} \nabla E\right)=\kappa_{\mathrm{R}} \rho[4 \pi B(T)-c E]+\sum_{n} S_{n}
$$

where $E$ is the radiation energy density, $\kappa_{\mathrm{R}}$ and $\kappa_{\mathrm{P}}$ are the Rosseland and Planck mean dust opacities, $\lambda$ is the flux-limiter, $\rho$ is the gas density, and $S_{n}$ are the stellar sources. This formulation of the diffusion equation omits velocity dependent terms that account for the advection of radiative enthalpy, since these are not significant for low-mass stars. At each timestep, the equation is solved iteratively until the temperature converges in each cell. The density is determined hydrodynamically and remains constant during the iterations. The opacities are time-lagged and also remain constant. We impose Marshak boundary conditions at the edge of the domain, which corresponds to a $10 \mathrm{~K}$ radiative flux. This ensures that the gas temperature limits to $10 \mathrm{~K}$ far from the stellar sources. Radiative diffusion is generally a good approximation within dense cores where the gas is fairly optically thick.

Figure 3 shows a comparison of the estimated dust temperatures around a young protostar. In both cases, the heating is mainly confined to the outflow cavity. In HYPERION, the difference between the core dust and outflow dust is due to radiative beaming. In ORION, although some beaming can occur (e.g., Cunningham et al. 2011), the elevated outflow temperature is due primarily to shocks. Consequently, these cells are a factor of $\sim 2$ warmer than estimated by HYPERION. Similar differences are apparent at later times.

\subsection{The Spectral Energy Distribution Zoo}

In this section we examine the evolution of the source SEDs as a function of time, resolution, aperture, and viewing angle.

\subsubsection{Resolution}

Figure 4 shows a source observed at each zooming stage. To allow structure to develop at each resolution,

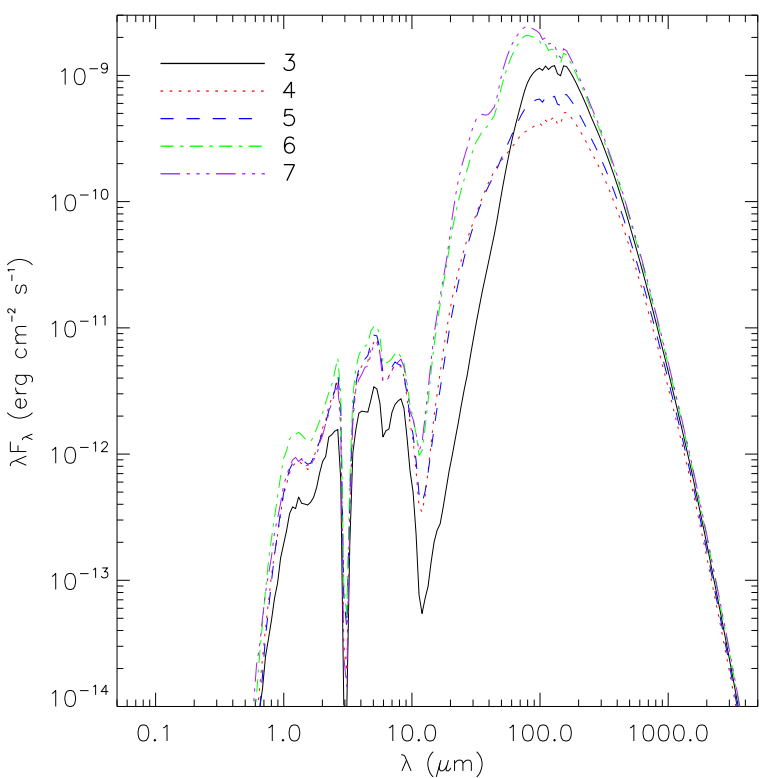

FIG. 4.- A $30 \mathrm{kyr}$ source with SEDs calculated at fixed inclination and $1000 \mathrm{AU}$ aperture. The curves range from low (maxlevel $=3,65 \mathrm{AU}$ ) to high (maxlevel=7, $4 \mathrm{AU}$ ) resolution. Consecutive SEDs are $\sim 1.5 \mathrm{kyr}$ apart.

there is an offset of $\sim 2000 \mathrm{kyr}$ between the zoom images. $^{2}$ For consistency, we impose an inner gas radius of $16 \mathrm{AU}$ (4 cells at the finest resolution zoom) for all zooms. At higher resolution, the main emission peak in the far-infrared shifts to shorter wavelengths. Progressively more emission escapes in the near-infrared as the central disk becomes more compact and the cavity extends closer to the star (see Section 3.3). However, the trends aren't entirely monotonic with resolution. At long wavelengths the SED is fully converged and emission comes from larger scales. Consequently, changes in the sub-millimeter portion of the SED partially result

2 New fine cells are initialized by interpolating data from the parent lower resolution cells. Without additional hydrodynamic evolution, the data on the finest level cannot be considered higher resolution. 


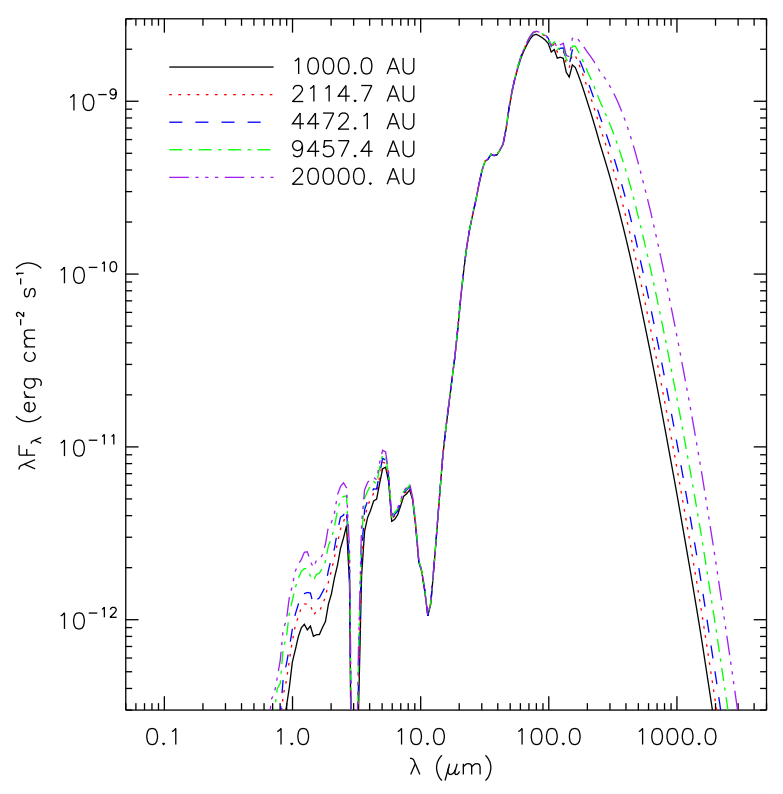

FIG. 5.- Source at $30 \mathrm{kyr}$ with SEDs at fixed inclination (same as in Figure 4) and at the maximum resolution viewed with five different aperture sizes.

from the time evolution between each resolution snapshot. The effects of time evolution and additional resolution cannot be disentangled.

\subsubsection{Aperture}

The choice of aperture can be observationally useful for probing emission at specific unresolved size scales. Since the long wavelength emission on larger scales primarily derives from the core envelope, emission differences between large and small apertures can be used to estimate the envelope gas mass. For multiple apertures, an average density profile can also be inferred by fitting how quickly the emission decreases with aperture size. Figure 5 illustrates the monotonically shrinking emission at long wavelengths as the aperture size decreases. The near-infrared emission also decreases slightly. This is due to the radiation emitted closer to the star scattering to larger scales.

\subsubsection{Time Evolution}

Star formation models suggest a basic progression in the source properties over time: the envelope mass decreases, the outflow cavity widens allowing more stellar radiation to escape, and the disk shrinks. However, exactly how this progression occurs and specifically how this impacts the SED in not well known. The R06 analytic prototypes for the source, disk, and envelope parameters encompass such changes broadly, without specifying an exact evolutionary sequence. Our simulated protostars, however, do follow a consistently calculated evolutionary progression. Asymmetric gas morphology and non-monotonic evolution, which results from effects such as variable stellar accretion, add another level of complication over the R06 models. In this section we consider the time evolution of the SEDs in our simulations and the effect on underlying properties.

Figure 6 shows the SEDs of the forming stars at four different times. The first forming star appears at all four times, while the second and third star appear at three times. In a few cases, there is a secondary or tertiary star present within the observing aperture. In these cases, the SEDs of the two sources are nearly identical, so we present only the SED for the most massive. Each panel shows the seds for the source viewed at four different inclinations (see section 4.5 for discussion). These SEDs correspond to the views with the highest and lowest bolometric luminosities and to two views near the median inferred bolometric luminosities.

Figure 6 illustrates several anticipated broad trends. The spectral peak shifts to shorter wavelengths with time. This shift is partially due to more direct and scattered emission escaping at shorter wavelengths. More viewing angles sample this emission with time (see section 3.2.4). Nearly all the sources at 60 kyr show some emission at shorter wavelengths, even the youngest sources. This is because star formation within the cloud is somewhat clustered, as illustrated by Figure 1, and the interacting outflows of the older sources impact the environment of the younger sources (see Figure 2).

For the sources with close companions, we find that the SED appearance is not directly indicative of a second nearby star. Without resolving the second source it is thus impossible to tell which of the sources have companions from the SED shapes alone. Disentangling the effects of multiplicity on the SED is an important problem, but it is beyond the scope of our current study.

Figure 7 shows the wavelength-integrated flux, i.e., luminosity, derived from the SEDs for each source and the range between the minimum and maximum. In some cases the range of luminosities spans an order of magnitude. For source 7, it spans three orders of magnitude. In almost all cases, the input luminosity falls within the observationally estimated range. In the three cases where there is large disagreement (sources 6, 11, 13), the observed protostars are near more luminous sources. Although none of these is physically within $1000 \mathrm{AU}$ of another source, the apertures may still overlap significantly with that of a brighter source, increasing the net emission. For example, source 6 lies $\sim 1300$ AU away from the brightest source, $1 \mathrm{~d}$. Since it is relatively close to $1 \mathrm{~d}$ and dim by comparison, it is unlikely that it would be identified as a separate source observationally.

Increasing the aperture size has two effects on the luminosity ranges shown in Figure 7 . First, as more envelope gas is included, the integrated luminosity increases. For large apertures, i.e., $\geq 10,000 \mathrm{AU}$, the minimum bolometric luminosity can be 2-3 times the actual protostellar luminosity (even accounting for the presence of a second protostar). The excess emission arises from the core envelope, which is radiating at $\sim 10 \mathrm{~K}$ (e.g., Enoch et al. 2008). Second, if a significant envelope remains, the IR flux contributes less to the total luminosity for large apertures such that the difference between the minimum and maximum bolometric luminosities decreases.

\subsubsection{Viewing Angle}

Previous comprehensive SED modeling has highlighted the influence of viewing angle on the inferred luminosity, SED shape, and apparent age (e.g., Whitney et al. 2003). We find a similar dependence here. Figure 6 illustrates SED variability with source inclination. For example, one view observes source 1c down the outflow 


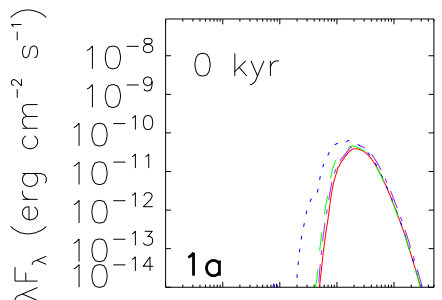

Classes

\begin{tabular}{|c|c|c|c|}
\hline $\operatorname{Max}$ & 1 & 2 & Min \\
\hline Early 0 & Early 0 & Early 0 & Early 0 \\
\hline Early 0 & Early 0 & Early 0 & rly \\
\hline Late I & Early 0 & Early 0 & arly \\
\hline Early I & Late 0 & Early 0 & arly \\
\hline Late 0 & Early 0 & Early 0 & Early \\
\hline Early 0 & Early 0 & Early 0 & Early \\
\hline Late 0 & Early 0 & Early 0 & Early \\
\hline Early I & Early 0 & Early 0 & Early \\
\hline Early 0 & Early 0 & Early 0 & Early \\
\hline Early 0 & Early 0 & Early 0 & Early \\
\hline Early I & Early 0 & Early 0 & Early \\
\hline Late 0 & Early 0 & Early 0 & arly \\
\hline Late 0 & Early 0 & Early 0 & arly \\
\hline Early 0 & Early I & Early I & Early \\
\hline Early I & Late 0 & Late 0 & Early \\
\hline Late 0 & Early I & Late 0 & Early \\
\hline Late 0 & Early I & Early 0 & arly \\
\hline Late 0 & Late 0 & Early 0 & rly \\
\hline Late 0 & Early 0 & Early 0 & Early \\
\hline Late 0 & Early I & Early 0 & $\|$ \\
\hline Early 0 & Early 0 & Early 0 & Early \\
\hline Early 0 & Early 0 & Early 0 & Early \\
\hline Early 0 & Early 0 & Early 0 & Early \\
\hline
\end{tabular}
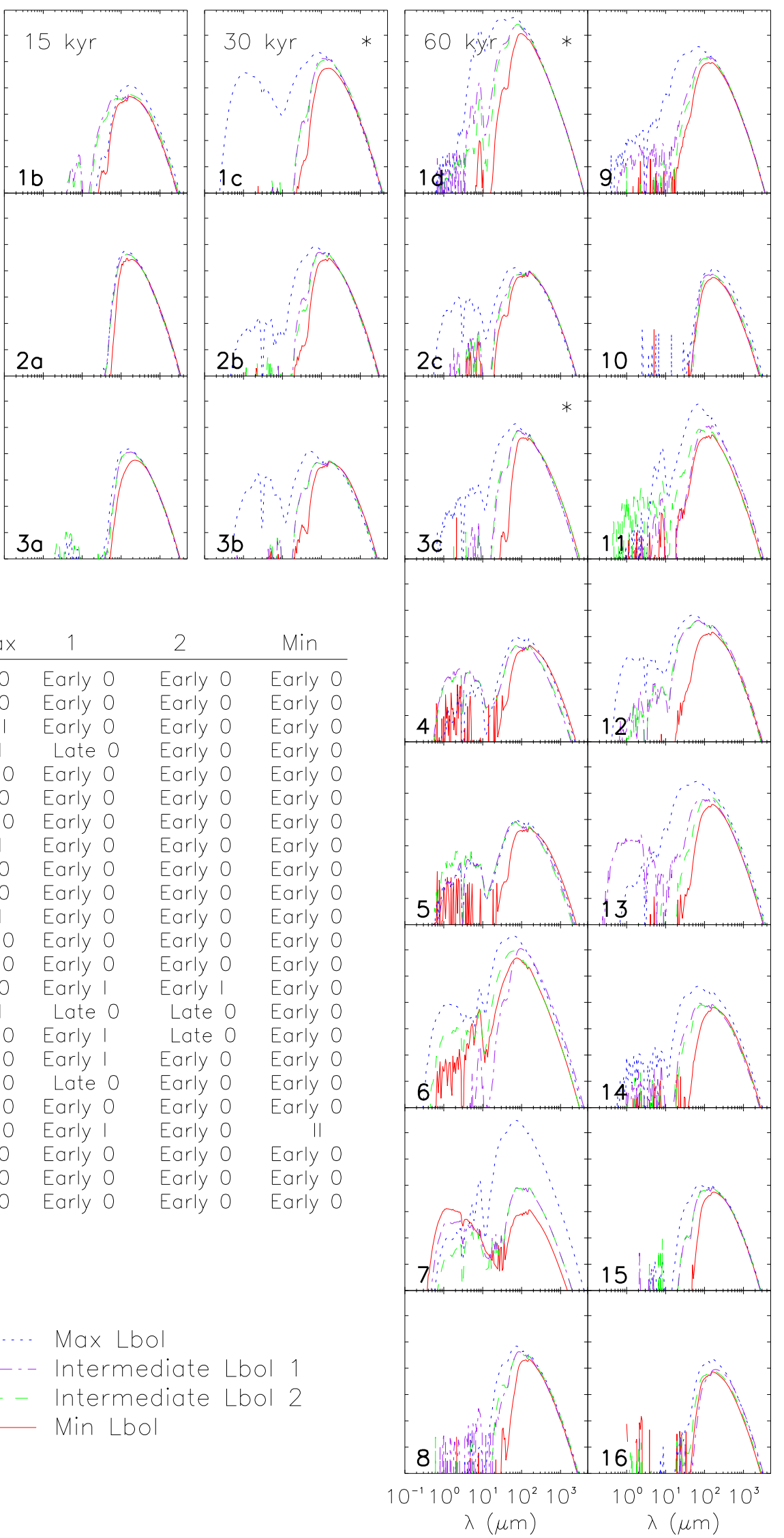

Fig. 6. - Sources at the four different times viewed through a 1000 AU radius aperture. The output time for each column is denoted in the first row. Each panel contains SEDs for four different viewing angles: the SEDs corresponding to the minimum and maximum bolometric luminosities and two intermediate bolometric luminosities. If there is a secondary protostar within 1000 AU only the SEDs for the primary source are depicted; these panels are marked with an asterisk. The inset table indicates the class of each SED. 


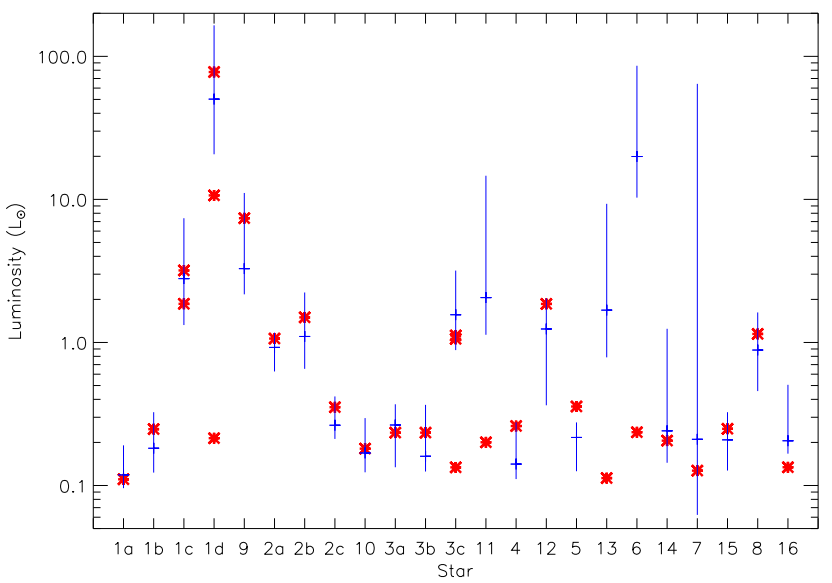

FIG. 7.- Bolometric luminosities for all sources shown in Figure 6. The input luminosity (simulation) for each model is indicated by the red stars. The vertical range indicates the bolometric luminosities spanned by the different views, where the 1st intermediate bolometric luminosity is marked by the horizontal line. The aperture radius is $1000 \mathrm{AU}$. The luminosities of sources within $1000 \mathrm{AU}$ of the primary are also shown (e.g., 1c, 1d, 3c).

cavity, making it appear much warmer and more evolved than its actual age of $\sim 35 \mathrm{kyr}$. In most cases, however, there is no clear sight line to the star since the outflow cavity is not entirely free of dust and gas. As a result, we find slightly less excess IR emission than idealized models for this view (e.g., Whitney et al. 2003).

Three of the sources (numbers 1, 2 and 3) are present during more than one zoom. The viewing angle corresponding to the SEDs with the minimum and maximum bolometric luminosities shown in Figure 6 is not necessarily the same across these panels. Since the sources are not fixed with respect to the grid, the orientation at which an observer would view the source, for example down the outflow cavity, evolves with time. However, the brightest and dimmest SEDs generally correspond to the views down the cavity and through the edge-on disk, respectively.

Figure 8 shows some typical luminosity distributions for 20 observed viewing angles. Younger more embedded sources tend to have a lower luminosity dispersion. This trend is apparent for source 1 in Figure 6. However, the picture becomes more confused when the sources are clustered. The environment of the younger protostars is contaminated by outflows and heating from older nearby objects.

\subsection{Protostellar Imaging}

The origin of the emission contributing to the SED shape is apparent in images of the constituent wave bands. Figure 9 shows two protostars viewed in six different wavelengths. The longer wavelengths pick out the colder envelope dust. In the mid-infrared, the cooler, edge-on disk becomes visible as a narrow extinction band perpendicular to the outflow. Towards shorter, nearinfrared wavelengths, scattered light highlights the outflow cavity. The radiative beaming caused by the outflow cavity is readily apparent. As sources become older and the dust warms, emission increases at shorter wavelengths. The images of the younger source, which is not edge-on, indicate a much narrower outflow cavity.

Figure 10 illustrates the effect of increasing the simula-

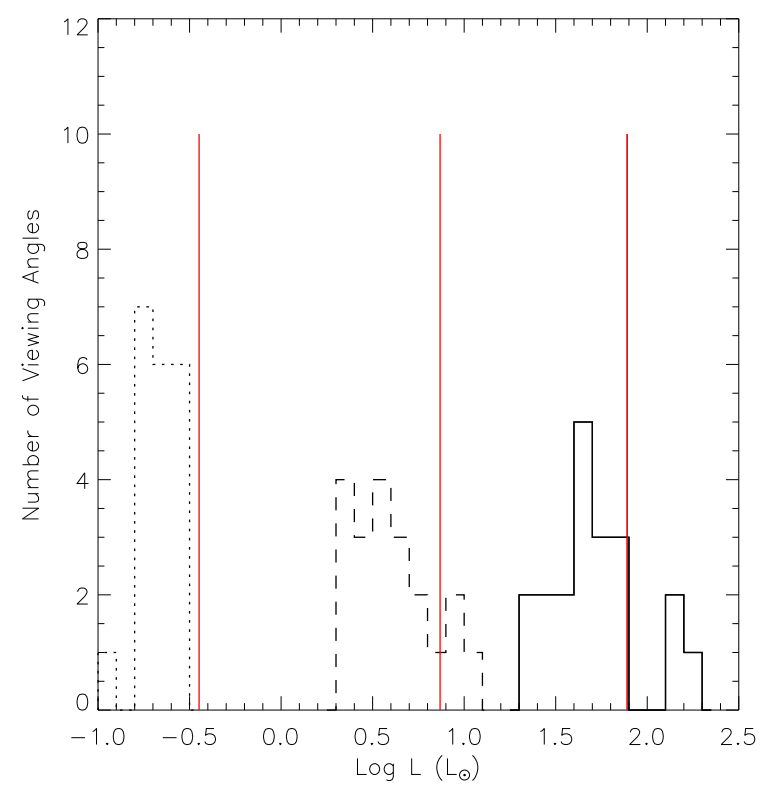

FIG. 8.- Histogram of bolometric luminosities for the 20 different viewing angles for sources 4 (left), 9 (middle) and 1d (right). The input luminosity (simulation) for each model is indicated by the red vertical lines. The aperture size is $1000 \mathrm{AU}$.

tion resolution. Overall, the higher resolution has a small effect on the outflow morphology. However, high density regions, such as the disk, become denser and more compact.

\subsection{Source Classification}

In this section, we compare the source evolutionary stage derived from observational metrics with the actual source properties. There are two main, arguably comparable, ways to assign a spectral Class to a source. The first involves computing the spectral slope of the SED in the near to mid IR (Greene et al. 1994). Young sources have a steeply increasing slopes $(\alpha \geq 0.3)$ that flatten and then decline $(\alpha<-1.6)$ as the source evolves and accretes its envelope. In this work, we use the characteristic spectral temperature, or "bolometric temperature", to determine the spectral Class.

The bolometric temperature, $T_{\mathrm{bol}}$, is defined as

$$
T_{\text {bol }}=1.25 \times 10^{-11}\langle\nu\rangle \mathrm{K},
$$

(Myers \& Ladd 1993) where the mean frequency is given by

$$
\langle\nu\rangle=\frac{\int \nu S_{\nu} d \nu}{\int S_{\nu} d \nu},
$$

where $S_{\nu}$ is the flux density. Low bolometric temperatures correspond to young, dim protostars with relatively massive, cold envelopes. The bolometric temperature gradually approaches the effective stellar surface temperature as the surrounding envelope is accreted or expelled. We separate the SEDs into early and late Class 0, early and late Class I, and Class II (e.g., Enoch et al. 2009; see Table 2 for definitions). The exact division of bolometric temperatures into classes is a matter of definition; in some cases $T_{\mathrm{bol}}=70 \mathrm{~K}$ serves as the dividing line between Class 0 and Class I objects (Enoch et al. 2009; Evans et al. 2009). Here we use $100 \mathrm{~K}$ as the cutoff between late Class 0 and early Class I objects. 


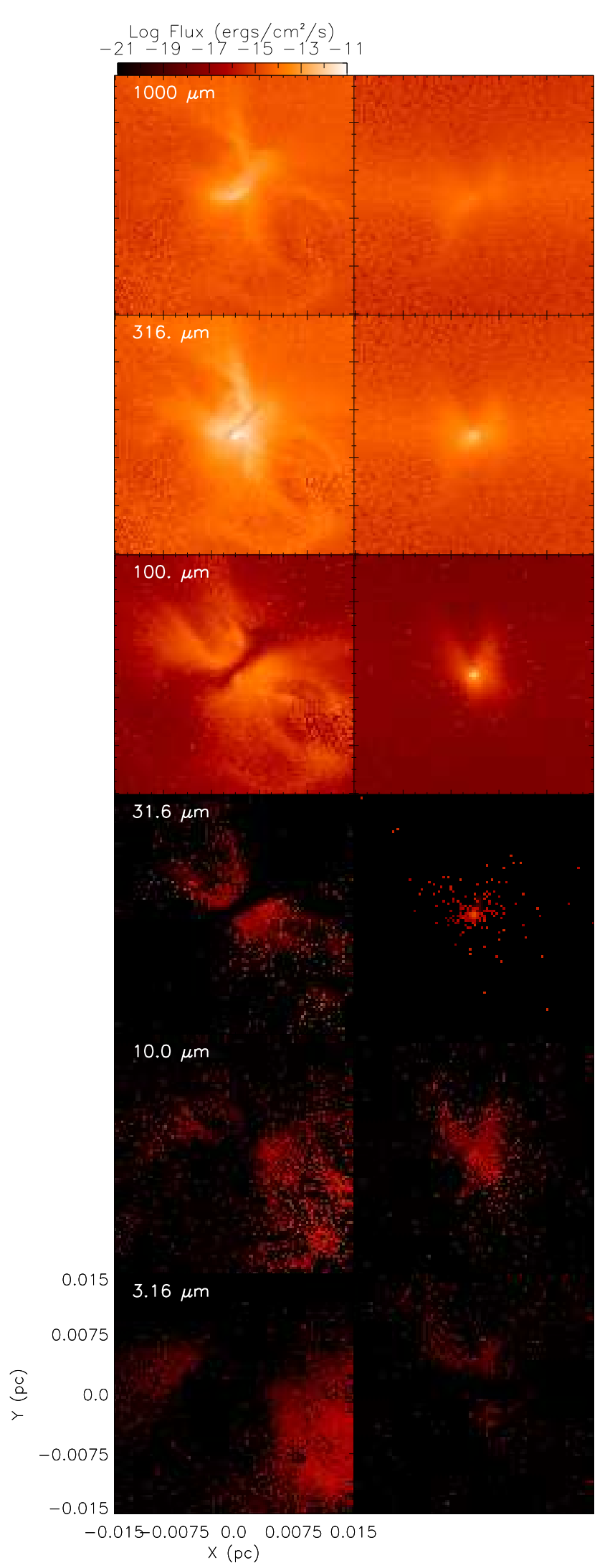

FIG. 9.- Two protostars in the $30 \mathrm{kyr}$ time slice imaged in six different wavebands. The first forming star in the simulation is on the left.

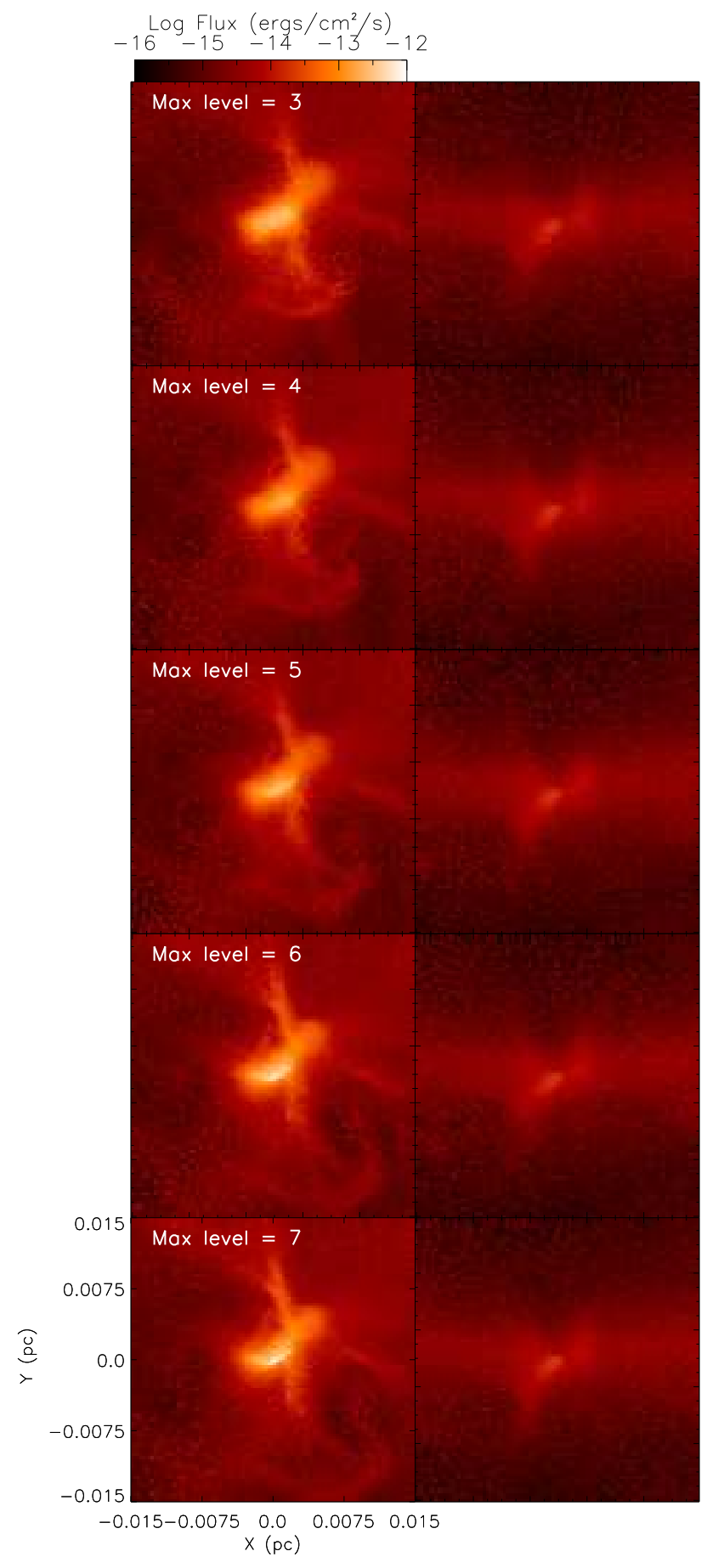

FIG. 10. - Two protostars in the $30 \mathrm{kyr}$ time slice (the first forming star is on the left) imaged in $1 \mathrm{~mm}$, where the AMR maximum resolution increases from $65 \mathrm{AU}$ (top) to $4 \mathrm{AU}$. The images are $~$ 1.5 kyr apart.

Figure 11 shows the inferred spectral Classes for several sources. The oldest (1c) shows a larger range of inferred Classes, including one view corresponding to late Class I. This indicates that even if a source is $\lesssim 30 \mathrm{kyr}$ old, which is below the estimated Class 0 lifetime of 40100 kyr (Enoch et al. 2009; Maury et al. 2011), it may appear as a Class I object $\sim 5-10 \%$ of the time. Statistically, some of these apparently older sources may be 
offset by older sources obscured by their disks, which appear younger. Reassuringly, the sources in Figure 11 appear to be early Class 0 from most inclinations. All the sources have a minimum bolometric temperature around $20 \mathrm{~K}$, which is reasonable given that the minimum dust temperature is $10 \mathrm{~K}$.

Table 2 summarizes the number of sources observed in each class at each time, assuming that every view corresponds to an independent observation. In reality, none of these sources are older than the typical estimated Class 0 lifetime, so it is surprising how many apparent Class I $(\sim 8 \%)$ and Class II $(\sim 1 \%)$ sources occur. If the estimated Class 0 lifetime were $\sim 70 \mathrm{kyr}$ or less and the star formation rate were constant, then we would expect equal numbers of early and late Class 0 sources when observing the cloud at the last output time. We find that $70 \%$ are early Class 0 sources, suggesting that the Class 0 lifetime is longer (although this argument implicitly assumes that the sources spend an equal amount of time in the early and late stages, which may not be the case).

In order to diminish the role of viewing angle on the classification, Crapsi et al. (2008) propose using a minimum envelope mass of $0.1 \mathrm{M}_{\odot}$ to distinguish between Class I (younger) and Class II (older) sources, i.e., between young embedded protostars (Stage I in the terminology of R06) and older disk-dominated sources (Stage II). We can estimate the envelope mass using

$$
M_{\mathrm{env}}=\frac{d^{2} S_{1 \mathrm{~mm}}}{B_{1 \mathrm{~mm}}\left(T_{\mathrm{D}}\right) \kappa_{1 \mathrm{~mm}}},
$$

where $d$ is the cloud distance, $S_{1 \mathrm{~mm}}$ is the flux density at $1.1 \mathrm{~mm}$ observed with a 30 " diameter aperture, $\kappa_{1 \mathrm{~mm}}=0.0114 \mathrm{~cm}^{2} \mathrm{~g}^{-1}$ is the dust opacity per gram of gas at $1.1 \mathrm{~mm}$ and $B_{1 \mathrm{~mm}}\left(T_{D}\right)$ is the Planck function evaluated at the dust temperature (Enoch et al. 2009). Applying this criterion to estimate the envelope mass for each source, we find that all the sources have envelopes exceeding $0.1 \mathrm{M}_{\odot}$ so that their evolutionary stage would be correctly identified as embedded protostars. The table illustrates the troubling problem of associating classes with physical stages. Some sources that are apparently Class I, and hence inferred to be older than the Class 0 lifetime of $\sim 0.1 \mathrm{Myr}$, are in fact much younger. Since the length of the Class 0 and Class I lifetimes are calculated statistically, for a large number of sources the effect of viewing angle will be minimized. However, the SEDs of individual sources may be misleading, and a fair fraction $(\sim 10 \%)$ of observed sources are inferred to be older than they actually are.

\section{COMPARISON WITH AN ANALYTIC MODEL GRID}

In this section, we compare the known simulated source properties with those inferred from fitting the R06 models to the SEDs. Rather than using the full spectral information, we interpolate the fluxes at commonly observed wavelengths. We include wavelengths 1.25, 1.65, $2.17 \mu \mathrm{m}$ to represent Two Micron All Sky Survey (2MASS) data, 3.6, 4.5, 5.8, $8.0 \mu \mathrm{m}$ to represent Spitzer IRAC, 24, 70, $160 \mu \mathrm{m}$ to represent Spitzer MIPS, and $1.1 \mathrm{~mm}$ representing data from the Bolocam continuum survey at the Caltech Sub-millimeter Observatory (CSO).

The R06 models assume a dust extinction ratio of $R_{v}=3.6$, which is slightly higher than what we adopt in our simulation and radiative post-processing. However,

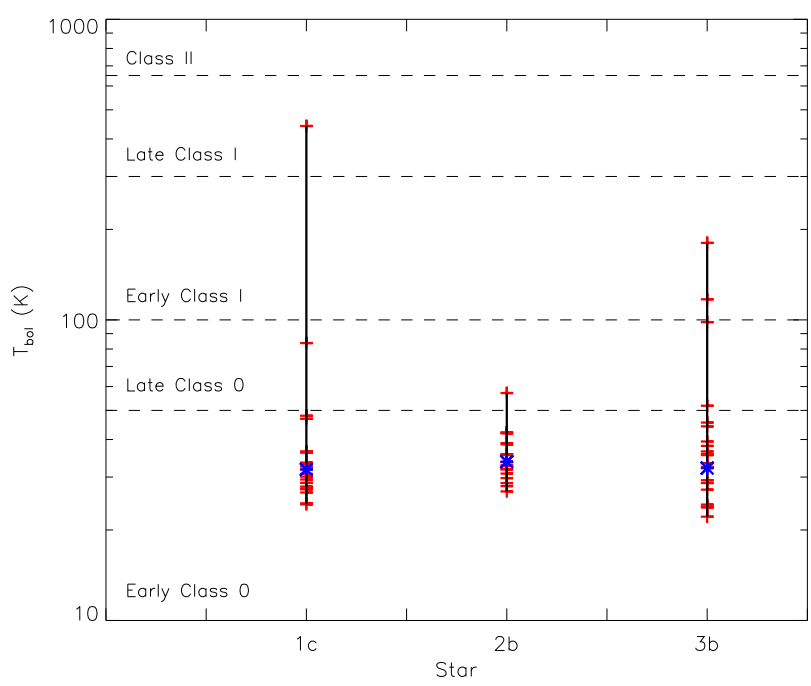

FIG. 11. - Bolometric temperatures and corresponding classification for three sources at $30 \mathrm{kyr}$ observed in a $1000 \mathrm{AU}$ radius aperture. The vertical bar indicates the range of values for 20 different views (red), while the blue star indicates the median value.

the R06 adopt different dust models for the disk than for the envelope and outflow. For the densest parts of the disks, R06 adopt the "disk midplane" model described by Whitney et al. (2003). This model has been shown to be a good fit for the SEDs of protoplanetary disks such as $\mathrm{HH} 30$ (Wood et al. 2002). For the remainder of the dust, R06 adopt the Kim et al. (1994) model, which has an average particle size only slightly larger than dust in the diffuse ISM. Neither model includes icy grain coatings, which are expected to increase the optical depth by a factor of $\sim 2$ (Chakrabarti \& McKee 2005). The former disk midplane model is more similar to the Semenov et al. (2003) model we use in ORION and in the HYPERION post-processing. The Semenov et al. (2003) model predicts an opacity 7.4 times larger at $1 \mathrm{~mm}$ and $\sim 2$ times larger at $0.1 \mathrm{~mm}$ than the R06 dust model. We discuss further in section 4.6 how opacity difference affects our results.

The R06 models include luminosity from the central star (Siess et al. 2000) and luminosity due to accretion from the disk onto the protostar:

$$
L_{\mathrm{acc}}=f_{\mathrm{acc}} \frac{G M_{*} \dot{M}_{*}}{R_{*}} .
$$

R06 adopts $f_{\text {acc }}=1$, which assumes that all the accretion energy is radiated away. In contrast, ORION adopts $f_{\text {acc }}=0.75$ to take into account that accretion may be radiatively inefficient and some energy may be advected into the star or drive a mass outflow (Ostriker \& Shu 1995).

In the following model comparison, we consider only those sources with "good" fits, which we define as $\chi^{2}-$ $\chi_{\text {best }}^{2}<3 N_{\text {data }}$, where $N_{\text {data }}$ is the number of flux data points considered. The model with the lowest $\chi^{2}$ given by $\chi^{2}<30 N_{\text {data }}$ is the "best-fit" model. In a few cases there is only a single model that satisfies these criteria, but in most cases there are a number of good-fit models. If a second source is within $1000 \mathrm{AU}$, we only analyze the model parameters for the primary. Figure 12 shows two sources with the corresponding best-fit models overlaid. 
TABLE 2

Number of PRotostars in EACH CATEGORY IF the ANGLE VIEWS ARE INDEPENDENT OBSERVATIONS.

\begin{tabular}{|c|c|c|c|c|c|}
\hline Time & $\begin{array}{l}\text { Early Class } 0 \\
T_{\text {bol }} \leq 50 \mathrm{~K}^{\mathrm{a}} \\
\end{array}$ & $\begin{array}{c}\text { Late Class } 0 \\
50 \mathrm{~K}<T_{\text {bol }} \leq 100 \mathrm{~K}\end{array}$ & $\begin{array}{c}\text { Early Class I } \\
100 \mathrm{~K}<T_{\text {bol }} \leq 300 \mathrm{~K}\end{array}$ & $\begin{array}{c}\text { Late Class I } \\
300 \mathrm{~K}<T_{\text {bol }} \leq 650 \mathrm{~K}\end{array}$ & $\begin{array}{c}\text { Class II } \\
650 \mathrm{~K}<T_{\text {bol }} \leq 2800 \mathrm{~K}\end{array}$ \\
\hline $0 \mathrm{kyr}$ & 20 & & & & \\
\hline $\begin{array}{l}15 \mathrm{kyr} \\
30 \mathrm{kyr}\end{array}$ & $\begin{array}{l}59 \\
53\end{array}$ & $\begin{array}{l}1 \\
4\end{array}$ & 2 & 1 & \\
\hline $60 \mathrm{kyr}$ & 233 & 59 & 22 & 3 & 3 \\
\hline
\end{tabular}
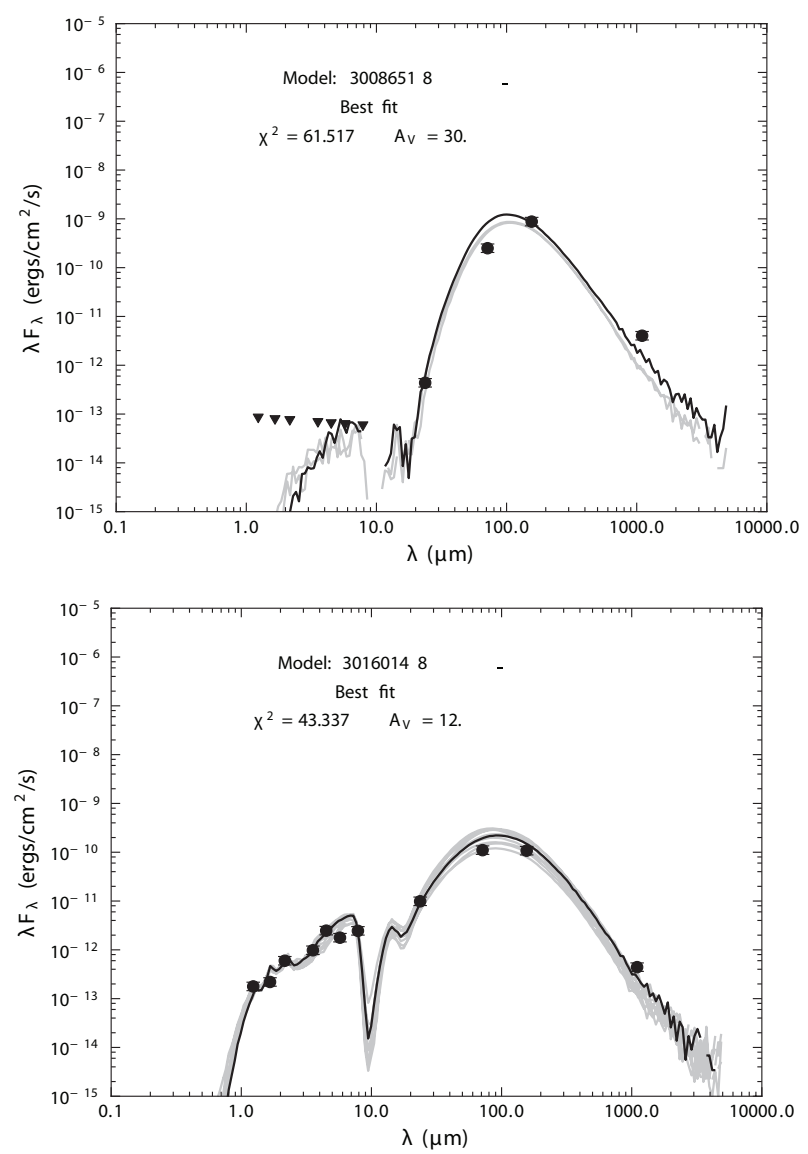

FIG. 12.- Observed fluxes in an $1000 \mathrm{AU}$ aperture for sources 1c (top) and 2c (bottom). Black points indicate the flux in the 2MASS, IRAC, MIPS, and BOLOCAM bands, where the triangles indicate upper limits imposed by the sensitivity of the instruments. The black line indicates the best-fit model, while the gray lines illustrate models with good $\chi^{2}$. The best $\chi^{2}$, dust extinction, scale, and best-fit model number are shown at the top.

The younger source (1c) has only upper limits for the 2MASS and IRAC bands. In general, we find that the models do not produce good fits for the most deeply embedded sources (e.g., all sources observed at 0 kyr and 15 kyr). This is partially due to "non-detections" in the IR, but also due to the source faintness. Two specific aspects of the R06 models make them unsuitable for fitting very young sources. First, the minimum model stellar mass is $0.1 \mathrm{M}_{\odot}$. In this case, the poor-fits are reassuring since the most massive star at $15 \mathrm{kyr}$ is $0.05 \mathrm{M}_{\odot}$. The second aspect is that the dust temperatures in the R06 models do not fall below $30 \mathrm{~K}$. Since the dimmest sources are surrounded by $10-20 \mathrm{~K}$ dust, they will naturally be colder than expected by the models.

Figures 13, 14, and 18 show the ratio of various model properties to the actual simulation values. Overall, the R06 models utilize more than 20 parameters (14 of which are independent) describing the protostellar source, disk, outflow, and envelope. Here, we restrict ourselves to comparing to eight fundamental parameters: stellar mass, stellar radius, stellar accretion rate, source inclination, envelope mass, disk mass, outer disk radius, and inner disk radius. The root mean square of the difference between the models and actual value are given in Table 3. We leave a more detailed comparison to future work. Also note that we compare each source for a single view (arbitrarily oriented with respect to the disk plane) and even though the source inclination is considered in the $\chi^{2}$ fit, the accuracy of the inferred parameters may depend upon the source orientation.

\subsection{Evolutionary Stage}

The primary physical quantity that can be derived from an SED is the evolutionary stage of the source. Since the R06 models cover a wide range of evolutionary stages, from embedded protostars to pre-main-sequence stars surrounded solely by low-mass disks, we first examine whether the models correctly identify that all the sources in the simulation are in the embedded protostellar evolutionary stage.

R06 defined three main stages of evolution based on the value of the stellar mass, $M_{*}$, and the envelope infall rate, $\dot{M}_{\text {env }}$. The latter is in fact a direct proxy for the envelope density, which is defined according to the rotationally flattened infalling envelope models of Ulrich (1976). In this classification scheme, Stage I sources (or envelopedominated sources) have $\dot{M}_{\text {env }} / M_{*} \geq 10^{-6} \mathrm{yr}^{-1}$, Stage II sources (disk-dominated sources with low-density or no circumstellar envelopes) have $\dot{M}_{\text {env }} / M_{*}<10^{-6} \mathrm{yr}^{-1}$ but $\dot{M}_{*} / M_{*} \geq 10^{-6} \mathrm{yr}^{-1}$, and Stage III sources (sources with little circumstellar material) have $\dot{M}_{\text {env }} / M_{*}<10^{-6} \mathrm{yr}^{-1}$ and $\dot{M}_{*} / M_{*}<10^{-6} \mathrm{yr}^{-1}$.

We find that all synthetic sources well fit by the R06 models have good-fit models with $\dot{M}_{\text {env }} / M_{*}>6 \times$ $10^{-5} \mathrm{yr}^{-1}$. Thus, the R06 models clearly indicate that the simulated sources are embedded Stage I sources. In other words, none of the models providing a good fit are disk-only models viewed at an extreme viewing angle.

Note that for the purposes of the model fitting there is no direct luminosity associated with $\dot{M}_{\text {env }}$; instead models with high values of $\dot{M}_{\text {env }}$ indicate the presence of a very dense envelope. The accretion rate associated with the output luminosity is $\dot{M}_{*}$, the rate at which mass is dumped from the disk onto the source (see comparison in section 4.4). For a realistic protostellar evolutionary sequence, there is likely a strong correlation between the infall rate and the stellar accretion rate. However, in the 
R06 model space these two rates are independent.

Physically, the infall rate may be many times higher than the stellar accretion rate if there is mass removal due to protostellar outflows and multiplicity (such that the infalling gas is distributed among two or more protostars). For example, Hansen et al. (2012) finds a core efficiency factor of $1 / 3$, which means that $2 / 3$ of the infalling core gas is expelled by outflows and thus $\dot{M}_{*}$ is correspondingly lower. In Offner et al. (2010), infall rates in radiation-hydrodynamic simulations without outflows fall in the range of $\sim 10^{-6} \mathrm{M}_{\odot} \mathrm{yr}^{-1}-6 \times 10^{-5} \mathrm{M}_{\odot} \mathrm{yr}^{-1}$. These are at least a factor of 2-20 times lower than the infall rates suggested by the R06 best-fit models, which predict $\sim 0.2-3 \times 10^{-4} \mathrm{M}_{\odot} \mathrm{yr}^{-1}$. If the cores are turbulent, as they are here, and not simply undergoing freefall collapse then the infall rate may be expected to be a factor of $\sim 2$ lower than suggested by the Ulrich (1976) model (McKee \& Tan 2003). Given these very high predicted infall rates, the stellar accretion rate must be significantly lower in order to avoid over-estimating protostellar luminosities. We will show that this is the case in section 4.4 .

\subsection{Stellar Mass}

Figure 13 illustrates that inferred masses tend to be more accurate for the older sources, where the true masses lie within or very near the model range (e.g., 1c6 ). The masses tend to be overestimated for the younger objects, which are somewhat contaminated by dust heated by neighboring protostars. Since the R06 models only include models with stellar masses $\geq 0.1 \mathrm{M}_{\odot}$, they will tend to over-estimate the masses of sources near this limit or below by construction. Overall, R06 bestfit models achieve the best accuracy for bright sources in non-clustered regions. However, only three of the 13 sources have best-fit models that disagree by more than a factor of 2. Given that the models span two orders of magnitude in source mass, the agreement between simulation and model is reasonable.

\subsection{Stellar Radius}

In contrast to the stellar mass, the R06 models systematically overestimate the stellar radius, generally by a factor of $\sim 2-3$. This is understandable since the R06 models use stellar properties interpolated from the nonaccreting isochrones of Siess et al. (2000). In contrast to Palla \& Stahler $(1991,1999)$, who agree well with the evolutionary model we adopt in ORION, the Siess et al. (2000) models do not include accretion. Instead, all protostars are initialized with their final stellar mass and a stellar structure determined by hydrostatic equilibrium for central temperatures $<10^{6} \mathrm{~K}$, i.e., pre-deuterium burning. Consequently, the Siess et al. (2000) protostars begin with inflated radii $>4 \mathrm{R}_{\odot}$ and contract towards the zero age main sequence. ${ }^{3}$ For Palla \& Stahler (1999), protostars with accretion rates of $10^{-5} \mathrm{M}_{\odot} \mathrm{yr}^{-1}$ terminate their accretion phase after $0.1 \mathrm{Myr}$ on an $L-T$ line in the H-R diagram referred to as the stellar birthline. This birth-line coincides fairly closely with the Siess et al. (2000) H-R placement of stars with ages of $0.1 \mathrm{Myr}$

\footnotetext{
${ }^{3}$ In the context of the R06 models, the "initial" radius is given at $10^{3}$ years, the earliest source age included in the models.
}

TABLE 3

Root Mean Square difference between the aCtual ORION AND R06 BEST-FIT VALUES FOR EACH PARAMETER AND THE MEDIAN RATIO OF THE BEST-FIT R06 AND ORION VALUES.

\begin{tabular}{lcc}
\hline \hline Parameter $^{\mathrm{a}}$ & $\mathrm{RMS}^{\mathrm{b}}$ & Median(R06/ORION) \\
\hline$M_{*}$ & $0.4 \mathrm{M}_{\odot}$ & 1.1 \\
$R_{*}$ & $2.8 \mathrm{R}_{\odot}$ & 2.4 \\
$d M_{*} / d t$ & $6.8 \times 10^{-7} \mathrm{M}_{\odot} \mathrm{yr}^{-1}$ & 2.2 \\
Inclination & $18.5 \mathrm{deg}$ & 0.7 \\
$M_{\text {env }}$ & $0.9 \mathrm{M}_{\odot}$ & 3.2 \\
$M_{\text {env }}, 4840 \mathrm{AU}$ & $57.0 \mathrm{M}_{\odot}$ & 6.2 \\
$M_{d}$ & $0.02 \mathrm{M}_{\odot}$ & 11.2 \\
$R_{d}$ & $174.1 \mathrm{AU}$ & 0.3 \\
$R_{d, \text { in }}$ & $27.6 \mathrm{AU}$ & 0.3 \\
\end{tabular}

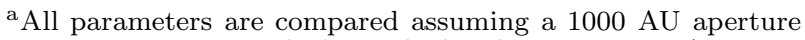
except $M_{\text {env }, 4840 \mathrm{AU}}$, which is calculated using a $4840 \mathrm{AU}$ aperture.

${ }^{\mathrm{b}}$ The RMS is defined as RMS $=\left(\sum_{i=1}^{N} \frac{\left[O_{i}-R 06_{i}\right]^{2}}{N}\right)^{1 / 2}$.

and masses $M<1 \mathrm{M}_{\odot}$ (e.g., Da Rio et al. 2010). Consequently, the two sets of models give similar values for the stellar radii as the stars approach 0.1 Myr. For stars with ages $\lesssim 0.05 \mathrm{Myr}$, the Siess et al. (2000) models overestimate the radii relative to our ORION model by a factor of $\sim 2$. Palla \& Stahler (1991) find that assuming lower accretion rates, e.g. $10^{-6} \mathrm{M}_{\odot} \mathrm{yr}^{-1}$, produces smaller prebirth-line radii. Thus, for protostars with low accretion rates and young ages, the two models will be even more discrepant. Some of the difference in model radii is offset by the ORION assumption that the accretion energy is not perfectly radiated away, i.e., $f_{\text {acc }}=0.75$. This results in an effective stellar radius of $\left(1 / f_{\text {acc }}\right) R_{*}$, which is somewhat closer to the estimated values of Siess et al. (2000)

Despite clear model differences, determining the absolute correctness of the stellar evolutionary models is not straight-forward. The initial protostellar radius, its subsequent evolution, and how it depends upon accretion and radiative efficiency, remains hotly debated (Hosokawa et al. 2011; Baraffe et al. 2009). Even without considering these effects, various calculations often adopt initial radii of $2.0 \mathrm{R}_{\odot}$ (Stahler 1988$)$ to $3.5 \mathrm{R}_{\odot}$ (Palla \& Stahler 1991). In cases where accretion is considered, accretion rates comparable to $\sim 10^{-6} \mathrm{M}_{\odot} \mathrm{yr}^{-1}$, which are appropriate for low-mass stars, tend to have early radii of $\sim 1.5 R_{\odot}$ (Palla \& Stahler 1991; Tan \& McKee 2004; Hosokawa \& Omukai 2009). Given the differences between stellar models, it is encouraging that many of the protostellar masses inferred are nonetheless quite close to the simulated values (see section 4.2 ).

\subsection{Stellar Accretion Rate}

The R06 models achieve fairly good agreement with $\dot{M}_{*}$. With the exception of two cases, the models either bracket the actual value or come very close to it. Since the good-fit models may span four orders of magnitude in the accretion rate, we plot the fourth root to reduce the error bar extent in the plot.

In the R06 models, the range of model accretion rates decreases monotonically with source age. If protostars experience episodic accretion (Hartmann \& Kenyon 


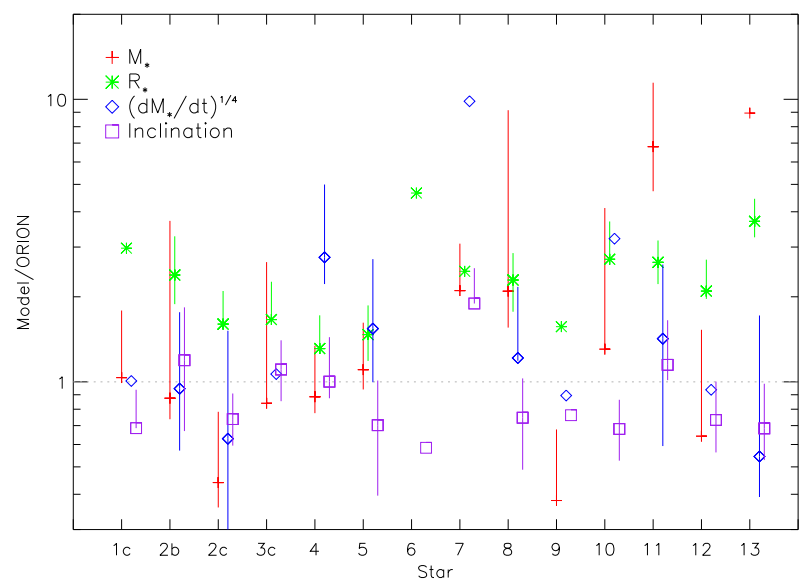

FIG. 13.- Ratio of the inferred best model values to the actual simulation value for each of the sources with good fits, where $M_{*}$ is the stellar mass, $R_{*}$ is the stellar radius, $\left(d M_{*} / d t\right)^{1 / 4}$ is the fourth root of the accretion rate onto the star, and the Inclination refers to the tilt with respect to the line of sight. The dotted line indicates where the models determine a value identical to the true value in the simulations. Points with no error bars have only one best fitting model parameter.

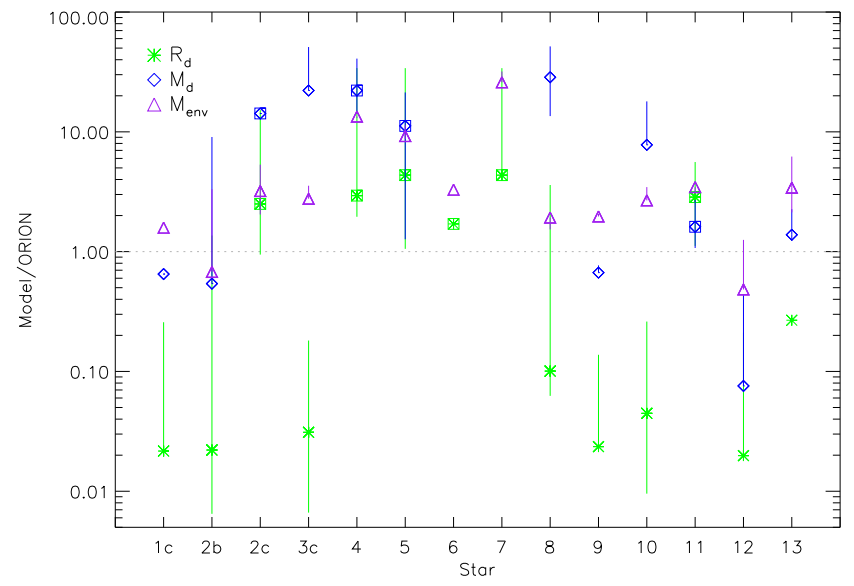

FIG. 14. - Ratio of the inferred best model values to the actual simulation value for each of the sources with good fits, where $M_{d}$ is the disk mass, $R_{d}$ is the stellar radius, and $M_{e n v}$ is the envelope mass. The dotted line indicates where the simulation and models are equivalent. Points with no error bars have only one best fitting model parameter. Boxed points indicate that the comparisons use the simulation upper limits for the disk mass or radius.

1996; Zhu et al. 2009; Vorobyov \& Basu 2010; Offner et al. $2011 b$ ), then protostellar accretion rates may jump between $10^{-7} \mathrm{M}_{\odot} \mathrm{yr}^{-1}$ and $10^{-4} \mathrm{M}_{\odot} \mathrm{yr}^{-1}$, extremes which may not be spanned by the models and thus result in age or mass errors. However, we find that, at least for these early times, variability in the instantaneous accretion rate is less than an order of magnitude. Consequently, our simulated values fall within the model ranges of R06. The extreme accretion events known as FU Ori outbursts are believed to result from a combination of thermal and magnetic instability in the inner accretion disk (Zhu et al. 2009). We do not treat these effects in these simulations, and thus all accretion variability is due to gravitational instability within the disk or turbulent variations of the core envelope.

The good agreement between the stellar accretion rate determined from the SED models and the accretion rate in the simulation is surprising, nonetheless, since the HYPERION source modeling assumes a single input spectrum with a bolometric luminosity equal to the sum of the stellar and accretion luminosities. Note that this sum is ultimately what sets the range of possible good-fit values for the protostellar masses and accretion rates shown in Figure 13. However, since there are many different values for the stellar and accretion luminosities that give the same bolometric luminosity, it should not be possible to disentangle the relative contributions from these from SED modeling. Instead, it is likely that the accretion rate is only constrained compared to the original range in the model grid due to other parameter constraints. In particular, due to the parameter space sampling in the R06 grid, models with higher envelope infall rates have higher stellar accretion rates, and conversely, models with lower envelope infall rates have lower stellar accretion rates (see Figure 1 of Robitaille 2008). Thus, constraining the envelope infall rate (or density), automatically restricts the stellar accretion rate to a range that is smaller than that of the whole set of models. Similarly, constraining the total luminosity also automatically rules out models with stellar accretion rates that are too high.

Figure 15 shows the distribution of stellar accretion rates for all models in the R06 set, for the models providing a good fit, and for all the models in R06 that have envelope infall rates and bolometric luminosities within $\pm 10 \%$ of the values of the good fits. ${ }^{4}$ The figure demonstrates that while the range of accretion rates for the good fits is much narrower than the range in the entire set of models, this range is primarily set by constraints on other parameters. It is therefore likely that $\dot{M}_{*}$ only appears to be well fit due to constraints on other parameters that can be more directly determined, such as the envelope infall rate and the bolometric luminosity. These two parameters supply the main constraints on the stellar accretion rate either by setting an upper bound (the luminosity) or by restricting the range for given envelope properties (the infall rate). However, we note that the agreement does appear to indicate that the accretion rates assumed in the $\mathrm{R} 06$ models span a realistic range.

\subsection{Inclination}

Inferring the correct source inclination is important for accurately determining other source properties. If a source is observed edge-on, but not identified by the models at this orientation, then it will appear erroneously younger. We use the simulated angular momentum of the protostar to determine the inclination with respect to the line of sight, where the net angular momentum vector is assumed to point perpendicular to the disk plane. Figure 13 illustrates that the most discrepancy occurs where the inclination is misfit, e.g., stars 8 and 9. These sources are inferred to have higher stellar masses and lower accretion rates than they actually do. At intermediate inclinations between pole-on and edge-on the inclination parameter is fairly degenerate. As a result, the inclination constraints are ultimately not very tight.

4 Since the infall rates and bolometric luminosites have been varied independently of the other fit parameters, these models are not necessarily close, i.e., within $10 \%$ of being good-fit models, to the data. 


\subsection{Envelope Mass}

For each R06 model, the envelope radius, $R_{\text {env }}$, is randomly drawn from a uniform logarithmic distribution of values between $R_{0} \times 4$ and $R_{0} / 4$, where $R_{0}$ is approximately the radius at which the optically thin radiative equilibrium temperature declines to $30 \mathrm{~K}$ :

$$
R_{0}=\frac{1}{2} R_{*}\left(\frac{T_{*}}{30 \mathrm{~K}}\right)^{2.5} .
$$

These values are further constrained to lie between $10^{3}$ AU and $10^{5} \mathrm{AU}$. Inside this radius, the density profile goes as $\rho(r) \propto r^{-3 / 2}$ with some slight modification due to rotational flattening. The envelope mass is then computed by integrating the mass inside $R_{\mathrm{env}}$. The ambient gas outside the envelope is assumed to be a constant density randomly drawn from the range 50 $\mathrm{cm}^{-3} \leq n_{\mathrm{H}_{2}} /\left(M_{*} / \mathrm{M}_{\odot}\right) \leq 100 \mathrm{~cm}^{-3}$. Thus, the envelope mass is coupled to both the density distribution and stellar properties.

In the simulations, however, the gas morphology is independent of the stellar properties and is not nicely spherically distributed. The mass asymmetry means that there is no clear cut way to define a physically meaningful envelope mass. Instead, we follow the R06 model convention and calculate the total mass inside a spherical volume with the radius $R_{\text {env }}$ suggested by the best fit model. This circumvents the need to define a density minimum or an effective radius for the simulations.

Figure 14 shows that the model envelope masses run high relative to the simulated envelope masses. In very few cases do the best fit models encompass the true value. This is primarily a result of the different opacity models adopted by ORION and R06. The envelope mass is determined from the long wavelength emission. At $1 \mathrm{~mm}$, the Semenov et al. (2003) opacity is 7.4 times higher than R06 dust opacity; it is $\sim 2$ times higher at $0.1 \mathrm{~mm}$. Since the envelope mass is inversely proportional to the opacity (see eq. 4.6), a given flux will be interpreted by the R06 models to correspond to a larger mass envelope. Figure 16 shows that the discrepancy is less for a 1000 AU aperture than for the larger apertures, which include more of the cold, extended envelope.

However, in all cases there is a fairly large scatter in the best-fit models. Since the simulated cores are embedded within a cloud and somewhat clustered, it is likely that some of the scatter is due to difficulty separating envelope emission from foreground emission, and thus correctly determining the line-of-sight extinction. The value of the inferred envelope radius is also important since most of the envelope mass is found a large radii. Since the R06 models only include envelope dust with temperatures $\gtrsim 30 \mathrm{~K}$, they preferentially neglect the coldest envelope mass. This can contribute to error in the $1 \mathrm{~mm}$ flux data point, which influences the determination of the envelope mass. Despite this innaccurary, the models do correctly identify the SEDs as belonging to embedded protostars and do not mistake them for more evolved disk-dominated sources.

For a given observed SED there will always be some underlying uncertainty in the grain composition and size distribution, which depends upon the local temperature, metallicity, and source age. However, our comparison suggests that without icy coatings and inclusion of cold

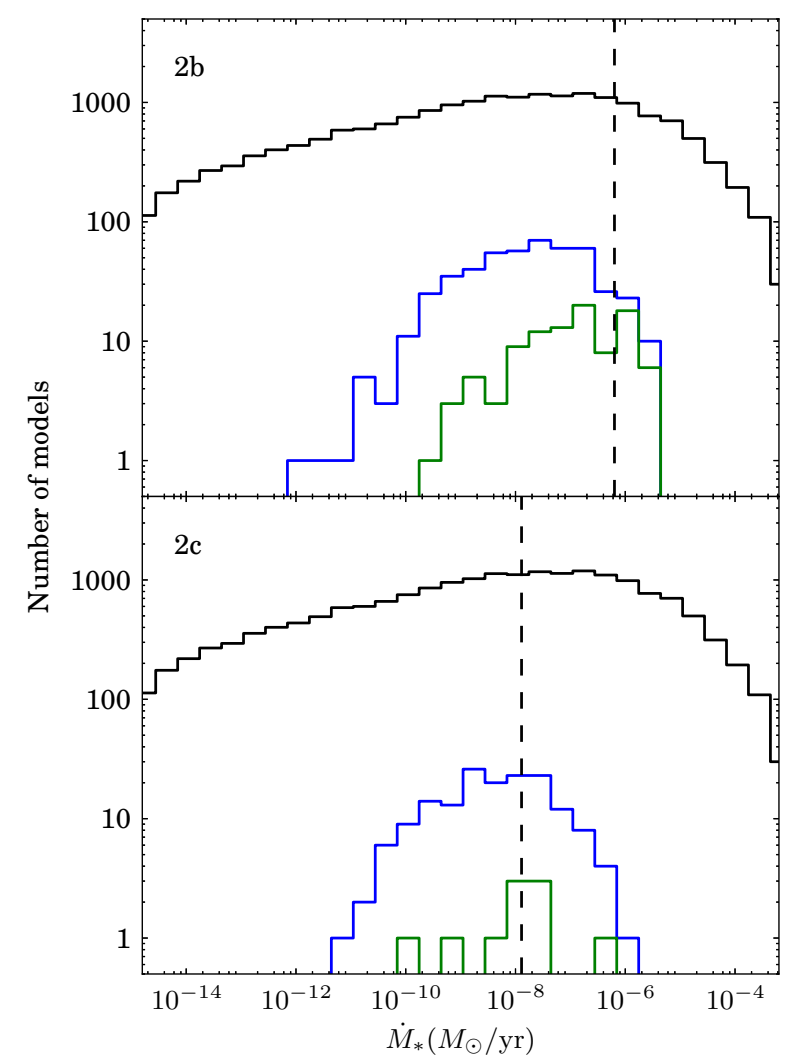

FIG. 15. - The top and bottom panels show, for two different sources, the distribution of stellar accretion rates, $\dot{M}_{*}$, in the entire R06 set of models (black), the models that provide a good fit to the SED (green), and the models in R06 that have envelope infall rates and bolometric luminosities within $\pm 10 \%$ of the values for each good fit. The vertical dashed lines indicate the best-fit model.

envelope gas, the R06 models may systematically overestimate observed envelope masses.

\subsection{Disk Mass}

We follow the same procedure as Offner et al. (2010) to define what constitutes a disk in the simulations. First, we identify all connected cells around the protostar with densities $\geq 10^{-15} \mathrm{~g} \mathrm{~cm}^{-3}$. This threshold effectively selects for gas comprising the disk, since the density of the gas in the envelope falls off rapidly. We then estimate the total angular momentum vector of the gas and rotate the coordinate frame so that the net angular momentum vector is parallel to the z-axis. Finally, we restrict the vertical disk height in the $\mathrm{z}$ direction to \pm 5 cells from the disk mid-plane. This is to eliminate confusion with streams of gas feeding the disk. The disk mass is then the integrated gas mass in these cells.

Due to the inclusion of outflows, we find that the disks are less orderly than in the simulations analyzed in Offner et al. (2010). In a few cases, i.e., where the protostellar mass is $\lesssim 0.1 \mathrm{M}_{\odot}$, there is no gas exceeding the density threshold. For these, we assume that the disk is too small to be resolved by the simulation and set the disk radius, $R_{d}$, to 4 fine cells (16 AU), the radius of the outflow launching region, and the disk mass to the mass enclosed in a spherical volume with this radius. These values thus represent upper limits on the disk mass and radius. 


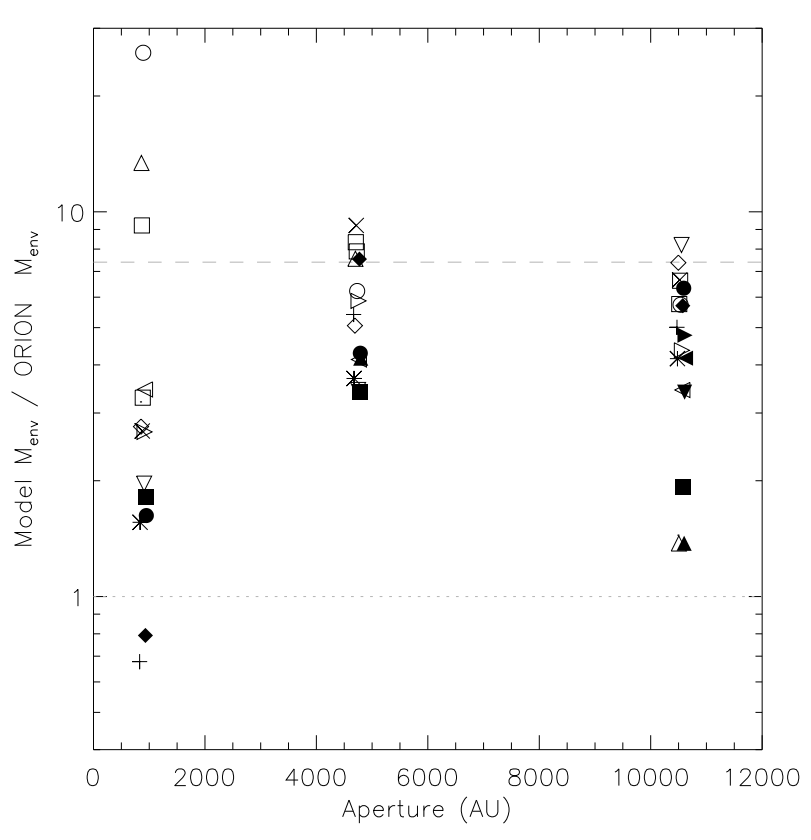

FIG. 16. - Ratio of the inferred best-fit envelope mass to the actual envelope mass for each of the sources with good fits as a function of aperture size. The dotted line indicates where the simulation and models are equivalent. The dashed line indicates the ratio of the ORION opacity to the R06 model opacity (7.4) at 1 $\mathrm{mm}$. All inferred envelope masses are shown (not only those centered on the most massive protostar in a multiple system). Each source has a unique symbol.

Figure 14 shows that the model disk masses are not well correlated with the simulated disk masses. The models tend to overestimate the disk mass, and in some cases the discrepancy is a factor of 10 . Unlike other parameters, such as the stellar mass, the actual disk mass does not fall within the range of good-fit models in nearly all cases. This is mainly because the simulated disks are more complex and less dense at the mid-plane, where the grid resolution does not resolve the scale height. The mass estimates are most overestimated in cases where the R06 model underestimate the size of the inner hole (see section 4.9), and thus overpredict the mass in the inner disk region. Since the simulated disks are more extended and less dense, it is also challenging for the models to accurately distinguish between emission from the disk and emission from the envelope, which contributes to the scatter in the agreement.

\subsection{Disk Radius}

In the R06 models, the disks are assumed to be perfectly symmetric with a sharp edge, so there is no difficulty in defining the disk radius. In the simulations, the disks are often asymmetric and occasionally have spiral structure that winds out to larger distances. For the simulations, we define the radius as the average radial distance of all the cells in the disk. This minimizes the effect of asymmetry on the radius estimate.

Figure 14 shows that the model disk radii are generally much smaller than the simulated disk radii. The bestfit model disks have a median radius of $\sim 32 \mathrm{AU}$ with a minimum value of $5.7 \mathrm{AU}$, while the simulations have a median of $\sim 136 \mathrm{AU}$ and minimum (upper limit) of 16 AU. This is to be expected since the simulations do not resolve the disk scale height and thus likely over estimate the disk extent. Since the disk mass is distributed over a larger volume and the gas is less dense than expected by the R06 models, some of the gas likely resembles envelope gas for the purpose of model fitting. However, the large range of radii inferred by the good-fit models suggests that this parameter is not well constrained by the SED in general. The sources where the model agrees within a factor of two are all comparisons assuming the upper limit of $16 \mathrm{AU}$ for the simulated disk radius. In principle, the disk properties should be sensitive to the viewing angle, however, we don't find that sources with better fit inclinations have necessarily more accurate disk properties. The accuracy of the inferred disk properties also likely depends on evolutionary stage. For more evolved sources, where the envelope mass is small, the R06 may determine more accurate disk masses and radii.

\subsection{Disk Inner Radius}

We choose to ignore the gas inside the accretion region, which corresponds to the $8^{3}$ cells centered on each star. This effectively enforces that all accretion disks have an inner radius, or hole, of $\sim 16$ AU. Since gas is removed from these inner cells during the accretion process at each timestep the dynamics in these cells is predominantly numerical. The outflow is launched just outside these cells so that gas remains close to the star when otherwise it might be evacuated by the outflow. To account for this, we set the density to zero in these cells when observing the protostars with HYPERION.

For Class 0 sources, which are heavily embedded, the details of the inner region have minimal effect on the SED characteristics. Figure 17 shows three SEDs calculated with and without the accretion region gas. Including the accretion gas produces an increased flux from $\sim 8-20 \mu \mathrm{m}$, i.e., increased photon scattering off of the inner warm dust. In the early Class 0 case (e.g., the green curve in Figure 17), the dust is optically thick to $<20 \mu \mathrm{m}$ emission and the inner region has negligible influence. The SEDs of late Class 0 and I sources are more sensitive to this emission. In Figure 17 the bolometric temperatures change by $3.6 \%$ (late Class 0 ) and $7.8 \%$ (late Class I), while the early Class 0 bolometric temperature only increases by $0.2 \%$. However, the difference turns out to have little effect on the properties inferred in the model comparison, which uses only discrete wavelength points from the SED. The largest flux difference falls between the observed $8 \mu \mathrm{m}$ and $24 \mu \mathrm{m}$ data points. Consequently, the inferred model properties are largely insensitive to the gas in the accretion region. (See Appendix A for additional discussion.)

We can verify that this is the case by comparing the inner disk radius inferred by the best fit models with the actual inner disk radius of 16 AU. The R06 models include disks with inner holes as large as $100 \mathrm{AU}$ in order to account for the possibility of a close, unresolved stellar companion that is evacuating the inner disk. Figure 18 shows the ratio of the inferred and actual inner disk radius. The large range of good-fit radii and large discrepancies of the best fit model suggest that the SED shape is not particularly sensitive to the inner hole size. At least for Class 0 objects, the accuracy is also insensitive to the magnitude of the bolometric temperature. 


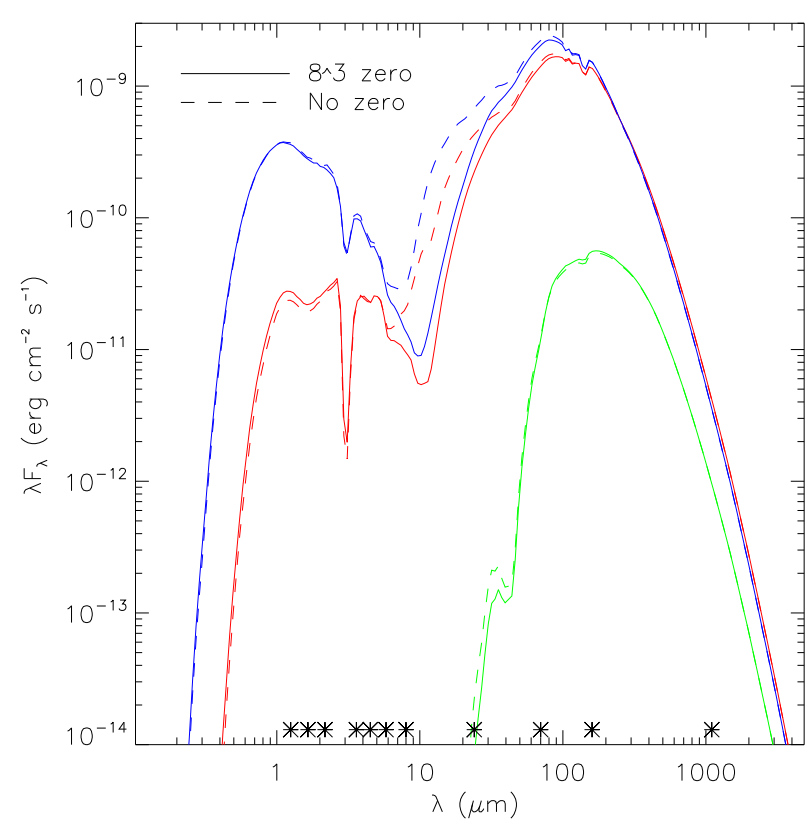

FIG. 17.- Three SEDs calculated with (dashed) and without (solid) the gas inside the accretion region. The three classifications are early Class 0 (green), late Class 0 (red) and late Class I (blue).

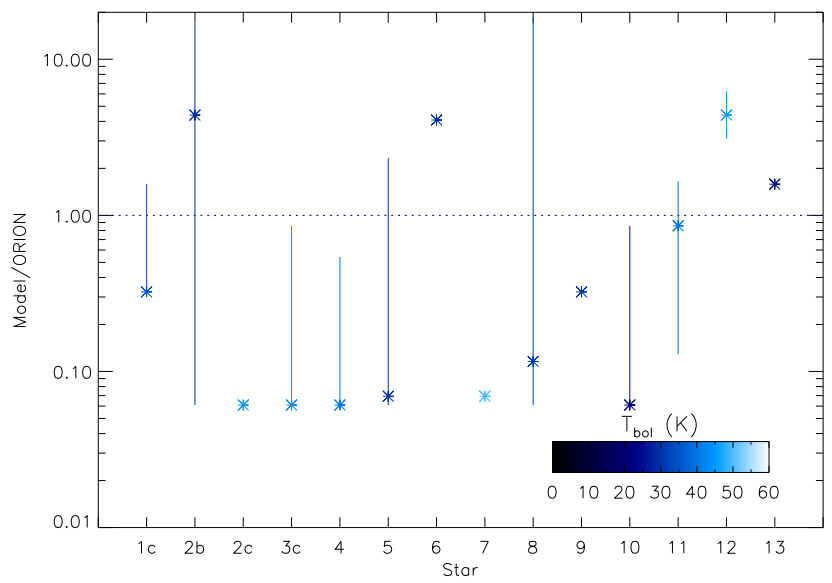

FIG. 18. - Ratio of the inferred best model inner disk radius to the actual simulation value for each of the sources with good fits. The dotted line indicates where the models determine a value identical to the true value in the simulations. Points with no error bars have only one best fitting model parameter. The color indicates the bolometric temperature.

\section{CONCLUSIONS}

We present results from turbulent, gravito-radiationhydrodynamics AMR simulations of forming stars. By including the effects of protostellar outflows and radiative heating, we are able to follow the evolution of the protostars self-consistently using realistic physics. We neglect magnetic fields, which we leave to be addressed in future work.

We use the Monte-Carlo radiative transfer code HYPERION to compute the synthetic spectral energy distributions of the sources for a variety of apertures, viewing angles, and resolutions. We find that the HYPERION and ORION calculated dust temperatures are most dis- crepant in shock heated gas such as within the outflow cavity, but generally agree within a factor of two for most cells.

Synthetic images of the forming protostars in the infrared and (sub) millimeter show clearly recognizable observational features. Emission from $\sim 1-30 \mu \mathrm{m}$ traces out the outflow cavity. In the sub-millimeter, an edge-on dense disk appears as a dark band obscuring the protostar.

Like previous work, we find that the source SED and its inferred properties are very sensitive to the viewing angle. The inferred bolometric luminosity variation can exceed a factor of 5 depending on the view. This corresponds to a typical standard deviation in log luminosity of 0.2. However, in most cases, the inferred range includes the true luminosity. The variety of SEDs also corresponds to a large range of bolometric temperatures, which can often span two spectral classifications. This underscores the difficulty of associating classes with specific evolutionary states based on SED characteristics alone. Since star formation is clustered, we find that the later forming stars suffer environmental contamination from the neighbors, increasing the uncertainties in their classification and inferred properties.

We next assess the accuracy of source properties inferred from analytic models. We use the model grid of Robitaille et al. (2006) to derive best-fit parameters for each of the sources and discuss the accuracy for a number of fundamental parameters. No good fits occur for the dimmest, most embedded objects, i.e., those with ages $<20$ kyr. The sources well fit by the R06 models are correctly identified as embedded protostars and not mistakenly fit with more-evolved disk-only models. Using the R06 models, we thus come to the same conclusion as when assessing the observed envelope mass: all the protostars are embedded Stage I objects. Since the R06 models do not include stars with final masses below $0.1 \mathrm{M}_{\odot}$, which is the critical envelope mass, there is small advantage to using the R06 models purely to determine the evolutionary stage in lieu of simply estimating the envelope mass. However, the R06 models offer many additional, potentially enlightening, parameter constraints. Further work extending the R06 model grid to lower masses may facilitate the evolutionary characterization of lower mass, young sources.

The model parameters determined from the good SED fits for the older sources exhibit varying amounts of accuracy compared to the true simulation parameters. We find that the stellar accretion rate spans the true value in most cases. The models are consistent with the true stellar mass for the older and/or more isolated sources. This is partially because the R06 models have a minimum mass of $0.1 \mathrm{M}_{\odot}$, and thus, will not well fit young low-mass sources by construction. In several cases, where the source environment is affected (via heating or outflow activity) by a more luminous neighbor, the stellar mass estimations are quite discrepant. The R06 models also use the Siess et al. (2000) stellar evolution models and thus systematically overestimate the source radii at early times. This may also introduce error into the parameter estimates. The inferred source inclination exhibits decent agreement with the actual inclination, although this parameter is not strongly constrained by the fits. The models systematically overestimate the envelope mass, 
a trend that might be corrected in future models that include icy grains and do not exclude the coldest envelope gas. We find that the disk mass and radius are not well fit by the models. While we expect that including magnetic effects and even better resolution would lead to smaller disks, ideally the breadth of the models should encompass the full disk parameter space. Some numerical studies have found that magnetic effects may completely suppress the formation of disks (Galli et al. 2006; Price \& Bate 2007; Hennebelle \& Ciardi 2009), which would also be challenging for the R06 models since they assume that all young sources have a disk.

Overall, our study highlights a number of problems with inferring source, disk, and envelope properties from observed SEDs of protostellar sources. Consequently, the classification and inferred properties of individual sources should be accepted with caution. Properties of isolated protostars are likely to be more accurate than protostars in clustered regions. As summarized in Table 3, properties such as the inferred stellar mass may be inaccurate by more than a factor of two and the envelope mass may be overestimated on average by a factor of four. The comparison is performed assuming that the sources are unresolved, so additional information from direct imaging might improve the accuracy of inferred parameters.

Some of our comparisons underscore uncertainty in fundamental star forming properties, such as dust properties and the dependence of stellar evolution on accre- tion, while others highlight improvements for future studies. A new and improved model grid using HYPERION will aim to improve some of these deficits (Robitaille et al. in prep). Although non-magnetized simulations show good agreement with observations (Bate 2009; Hansen et al. 2012) and we find that the resolution of the inner region has a small affect on our results, future numerical studies including magnetic fields and sub-AU resolution will be advantageous.

The authors thank Andrew Cunningham for helpful discussions of protostellar outflows. This research has been supported by the NSF through grants AST-0901055 (SSRO) and AST-0908553 (CFM and RIK), by NASA through the Spitzer Space Telescope Fellowship Program (TPR) and ATFP grant NNX09AK31G (RIK, CFM), and by the US Department of Energy at LLNL under contract DE-AC52-07NA (RIK). The ORION simulations were performed on resources managed by the National Energy Research Scientific Computing Center, which is supported by the Office of Science of the U.S. Department of Energy under Contract No. DE-AC0205CH11231. The HYPERION calculations and other data analysis were performed on the Odyssey cluster, which is supported by the Harvard FAS Sciences Division Research Computing Group. Figure 2 was rendered using yt (Turk et al. 2011).

\section{REFERENCES}

Baraffe, I., Chabrier, G., \& Gallardo, J. 2009, ApJ, 702, L27

Bate, M. R. 2009, MNRAS, 392, 1363

-. 2011, MNRAS, 2080

Chakrabarti, S. \& McKee, C. F. 2005, ApJ, 631, 792

Crapsi, A., van Dishoeck, E. F., Hogerheijde, M. R., Pontoppidan, K. M., \& Dullemond, C. P. 2008, A\&A, 486, 245

Cunningham, A. J., Klein, R. I., Krumholz, M. R., \& McKee, C. F. 2011, ArXiv e-prints

Da Rio, N., Robberto, M., Soderblom, D. R., Panagia, N., Hillenbrand, L. A., Palla, F., \& Stassun, K. G. 2010, ApJ, 722, 1092

Duchêne, G., Delgado-Donate, E., Haisch, Jr., K. E., Loinard, L., \& Rodríguez, L. F. 2007, Protostars and Planets V, 379

Enoch, M. L., Evans, N. J., Sargent, A. I., \& Glenn, J. 2009, ApJ, 692, 973

Enoch, M. L., Evans, II, N. J., Sargent, A. I., Glenn, J., Rosolowsky, E., \& Myers, P. 2008, ApJ, 684, 1240

Espaillat, C., Furlan, E., D'Alessio, P., Sargent, B., Nagel, E., Calvet, N., Watson, D. M., \& Muzerolle, J. 2011, ApJ, 728, 49

Evans, N. J., Dunham, M. M., Jørgensen, J. K., Enoch, M. L., Merín, B., van Dishoeck, E. F., Alcalá, J. M., Myers, P. C., Stapelfeldt, K. R., Huard, T. L., Allen, L. E., Harvey, P. M., van Kempen, T., Blake, G. A., Koerner, D. W., Mundy, L. G., Padgett, D. L., \& Sargent, A. I. 2009, ApJS, 181, 321

Galli, D., Lizano, S., Shu, F. H., \& Allen, A. 2006, ApJ, 647, 374

Greene, T. P., Wilking, B. A., Andre, P., Young, E. T., \& Lada C. J. 1994, ApJ, 434, 614

Hansen, C. E., Klein, R. I., McKee, C. F., \& Fisher, R. T. 2012, ApJ, 747, 22

Hartmann, L. \& Kenyon, S. J. 1996, ARA\&A, 34, 207

Heiderman, A., Evans, II, N. J., Allen, L. E., Huard, T., \& Heyer, M. 2010, ApJ, 723, 1019

Hennebelle, P. \& Ciardi, A. 2009, A\&A, 506, L29

Hosokawa, T., Offner, S. S. R., \& Krumholz, M. R. 2011, ApJ, 738, 140

Hosokawa, T. \& Omukai, K. 2009, ApJ, 691, 823

Kang, M., Bieging, J. H., Povich, M. S., \& Lee, Y. 2009, ApJ, 706 , 83

Kim, S.-H., Martin, P. G., \& Hendry, P. D. 1994, ApJ, 422, 164

Klein, R. I. 1999, Journal of Computational and Applied Mathematics, 109, 123
Krumholz, M. R., McKee, C. F., \& Klein, R. I. 2004, ApJ, 611, 399

Lada, C. J. 2006, ApJ, 640, L63

Lucy, L. B. 1999, A\&A, 344, 282

Matzner, C. D. \& McKee, C. F. 1999, ApJ, 526, L109

Maury, A. J., André, P., Men'shchikov, A., Könyves, V., \& Bontemps, S. 2011, A\&A, 535, A77

McKee, C. F. \& Offner, S. S. R. 2010, ApJ, 716, 167

McKee, C. F. \& Tan, J. C. 2003, ApJ, 585, 850

Myers, P. C. \& Ladd, E. F. 1993, ApJ, 413, L47

Offner, S. S. R., Capodilupo, J., Schnee, S., \& Goodman, A. A. 2011a, ArXiv e-prints

Offner, S. S. R., Klein, R. I., McKee, C. F., \& Krumholz, M. R. 2009, ApJ, 703, 131

Offner, S. S. R., Kratter, K. M., Matzner, C. D., Krumholz, M. R., \& Klein, R. I. 2010, ApJ, 725, 1485

Offner, S. S. R., Lee, E. J., Goodman, A. A., \& Arce, H. 2011b, ApJ, 743, 91

Offner, S. S. R. \& McKee, C. F. 2011, ApJ, 736, 53

Ostriker, E. C. \& Shu, F. H. 1995, ApJ, 447, 813

Palla, F. \& Stahler, S. W. 1991, ApJ, 375, 288

-. 1999, ApJ, 525, 772

Price, D. J. \& Bate, M. R. 2007, Ap\&SS, 311, 75

Robitaille, T. P. 2008, in Astronomical Society of the Pacific Conference Series, Vol. 387, Massive Star Formation: Observations Confront Theory, ed. H. Beuther, H. Linz, \& T. Henning, 290

Robitaille, T. P. 2011, ArXiv e-prints

Robitaille, T. P. \& Whitney, B. A. 2010, ApJ, 710, L11

Robitaille, T. P., Whitney, B. A., Indebetouw, R., \& Wood, K. 2007, ApJS, 169, 328

Robitaille, T. P., Whitney, B. A., Indebetouw, R., Wood, K., \& Denzmore, P. 2006, ApJS, 167, 256

Semenov, D., Henning, T., Helling, C., Ilgner, M., \& Sedlmayr, E. 2003, A\&A, 410, 611

Siess, L., Dufour, E., \& Forestini, M. 2000, A\&A, 358, 593

Stahler, S. W. 1988, ApJ, 332, 804

Stone, J. M., Ostriker, E. C., \& Gammie, C. F. 1998, ApJ, 508, L99

Swift, J. J. \& Welch, W. J. 2008, ApJS, 174, 202

Tan, J. C. \& McKee, C. F. 2004, ApJ, 603, 383 


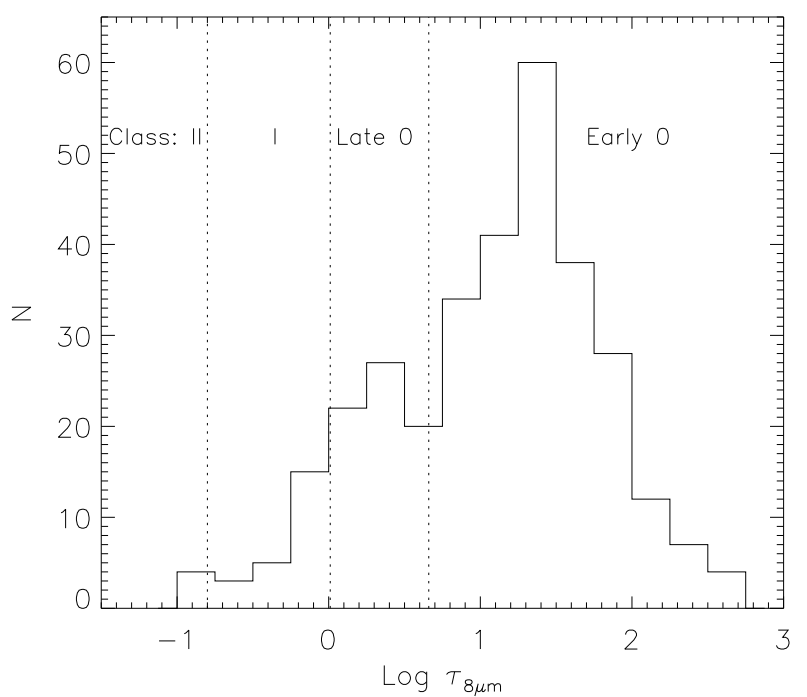

FIG. A1. - Distribution of optical depths at $8 \mu \mathrm{m}$ for each of the viewing angles for the sources at 60 kyr. The vertical lines indicate the approximate divisions between classes (see Table 2).

Truelove, J. K., Klein, R. I., McKee, C. F., Holliman, II, J. H. Howell, L. H., \& Greenough, J. A. 1997, ApJ, 489, L179+

Truelove, J. K., Klein, R. I., McKee, C. F., Holliman, II, J. H., Howell, L. H., Greenough, J. A., \& Woods, D. T. 1998, ApJ, 495,821

Turk, M. J., Smith, B. D., Oishi, J. S., Skory, S., Skillman, S. W., Abel, T., \& Norman, M. L. 2011, ApJS, 192, 9

Ulrich, R. K. 1976, ApJ, 210, 377

van Kempen, T. A., van Dishoeck, E. F., Salter, D. M., Hogerheijde, M. R., Jørgensen, J. K., \& Boogert, A. C. A. 2009, A\&A, 498, 167
Vorobyov, E. I. \& Basu, S. 2010, ApJ, 719, 1896

Whitney, B. A., Wood, K., Bjorkman, J. E., \& Wolff, M. J. 2003, ApJ, 591, 1049

Wood, K., Lada, C. J., Bjorkman, J. E., Kenyon, S. J., Whitney, B., \& Wolff, M. J. 2002, ApJ, 567, 1183

Zhu, Z., Hartmann, L., Gammie, C., \& McKinney, J. C. 2009, ApJ, 701,620

APPENDIX

\section{SENSITIVITY OF THE RESULTS TO THE INNER DISK RESOLUTION}

With or without the inclusion of the accretion region gas, the inner disk properties are not well resolved even with $4 \mathrm{AU}$ cell resolution. Observationally, the warm inner disk gas contributes SED emission at $\leq 8 \mu \mathrm{m}$ wavelengths (Robitaille et al. 2006). In observed SEDs, this emission can be used to directly infer disk properties (e.g., disk mass, flaring, gap size) of non-embedded protostars (e.g., Espaillat et al. 2011). In instances where the protostar is deeply embedded, namely during the Class 0 phase, radiation from gas close to the star is significantly reprocessed by the cold, envelope gas. As we have demonstrated in section 4.9 the properties inferred by the R06 models are relatively insensitive to the details of the inner disk in most cases.

We can estimate the importance of the inner regions more quantitatively by estimating the $8 \mu \mathrm{m}$ optical depth for different protostellar views. In cases where the optical depth $\tau_{8} \lesssim 1$, our models will not accurately represent the SED at short wavelengths. For $\tau_{8} \sim$ a few, the gas is optically thick to the emission from the inner regions, and the SEDs will not be significantly effected by the details of the numerical simulations on these size scales.

Figure A1 shows the distribution of optical depths at $8 \mu \mathrm{m}$ for all the different views of the protostars at the final output time. Only 27 views have optical depths $<1$. This approximately corresponds to the number of Class I and II sources that we find. Since these objects are viewed on sight-lines down the outflow cavity, we would a priori expect a low optical depth. The large majority of sources are Class 0. Based upon these optical depths, we expect that our simulated early Class 0 and most late Class 0 SEDs accurately represent real SEDs. The simulated SEDs of later classes will only accurately represent protostellar disks with large inner holes. Our results in section 4, which are obtained for Class 0 sources, should thus be generally applicable and relevant to observations. 